Pacific

Journal of

Mathematics

EXISTENCE OF SOLUTIONS AND REGULARITY

NEAR THE CHARACTERISTIC BOUNDARY

FOR SUB-LAPLACIAN EQUATIONS ON CARNOT GROUPS

DiMITER VASSILEV

Volume $227 \quad$ No. 2

October 2006 


\title{
EXISTENCE OF SOLUTIONS AND REGULARITY NEAR THE CHARACTERISTIC BOUNDARY FOR SUB-LAPLACIAN EQUATIONS ON CARNOT GROUPS
}

\author{
Dimiter VASSILEV
}

\begin{abstract}
We prove that the best constant in the Folland-Stein embedding theorem on Carnot groups is achieved. This implies the existence of a positive solution of the Yamabe-type equation on Carnot groups. The second goal of the paper is to show a certain regularity of the Green's function and solutions of the Yamabe equation involving the sub-Laplacian near the characteristic boundary of a domain in the considered groups.
\end{abstract}

\section{Introduction}

In this paper we consider problems of existence and regularity of solutions to a nonlinear Dirichlet problem involving sub-Laplacians on Carnot groups. The main motivation comes from the classical Yamabe problem, the question of determining the best constant in the Sobolev embedding inequality, and their CR counterparts.

A Carnot group is a simply connected and connected Lie group $\boldsymbol{G}$, whose Lie algebra $\mathfrak{g}$ admits a stratification $\mathfrak{g}=\bigoplus_{j=1}^{r} V_{j}$ with

$$
\left[V_{1}, V_{j}\right]=V_{j+1} \quad \text { for } 1 \leq j<r, \quad\left[V_{1}, V_{r}\right]=\{0\} .
$$

Let $Q=\sum_{j=1}^{r} j \operatorname{dim} V_{j}$ be the homogeneous dimension.

Our starting point is this embedding result of Folland and Stein, [Folland 1975]: For any $p \in(1, Q)$ there exists $S_{p}=S_{p}(\boldsymbol{G})>0$ such that, for $u \in C_{0}^{\infty}(\boldsymbol{G})$,

$$
\left(\int_{\boldsymbol{G}}|u|^{p^{*}} d H\right)^{1 / p^{*}} \leq S_{p}\left(\int_{\boldsymbol{G}}|X u|^{p} d H\right)^{1 / p} .
$$

Here, the horizontal gradient $|X u|$ is defined as $|X u|=\left(\sum_{j=1}^{m}\left(X_{j} u\right)^{2}\right)^{1 / 2}$, where $X=\left\{X_{1}, \ldots, X_{m}\right\}$ is a basis of $V_{1}, p^{*}=p Q /(Q-p)$, and $d H$ is a fixed Haar measure on $\boldsymbol{G}$.

Unlike the Euclidean case [Talenti 1976; Aubin 1976b], the value of the best possible constant or the nonnegative functions for which it is achieved is unknown.

MSC2000: $35 \mathrm{~J} 70$.

Keywords: subelliptic regularity, Sobolev embedding, Carnot groups. 
In the particular case when $p=2$ and $\boldsymbol{G}=\mathbb{R}^{n}$, this problem is related to the Yamabe problem ([Trudinger 1968; Aubin 1976a; Schoen 1984], see also the survey article [Lee and Parker 1987]); in the case of the Heisenberg group, it is related to the CR Yamabe problem. The case of odd-dimensional spheres is equivalent to the problem of determining the best constant in the $L^{2}$ Folland-Stein inequality on the Heisenberg group, and the functions for which it is achieved. The solution of this problem is instrumental for solving the general case, which is complete after the works of Jerison and Lee $[1984 ; 1987 ; 1988 ; 1989]$, and Gamara and Yacoub [Gamara 2001; Gamara and Yacoub 2001].

We note that, when $p=2$, the Yamabe equation is the Euler-Lagrange equation satisfied by the extremals of the naturally associated variational problem. It is also of interest to study the Yamabe equation on sets different from the whole group, especially in connection with certain blow-up arguments that appear, for example, in questions of existence of solutions on domains with a nontrivial topology [Bahri and Coron 1988; Brezis 1986; Citti and Uguzzoni 2001].

We consider similar problems in a general Carnot group. The method of concentration compactness of P. L. Lions can be used, and we show in Section 3 that there exists a best constant in the Folland-Stein inequality. It is achieved on the space $\mathscr{\mathscr { D }}^{1, p}(\boldsymbol{G})$, which is the closure of $C_{0}^{\infty}(\boldsymbol{G})$ with respect to the norm

$$
\|u\|_{\mathfrak{I}^{1}, p(\boldsymbol{G})}=\left(\int_{\boldsymbol{G}}|X u|^{p} d H\right)^{1 / p} .
$$

This method does not allow an explicit determination of the best constant or the functions for which it is achieved. The problem can be formulated as a variational problem. We consider the case $p=2$ in detail. The Euler-Lagrange equation of the nonnegative extremals leads to the Yamabe-type equation

$$
\mathscr{L} u=-u^{2^{*}-1},
$$

where $\mathscr{L} u=\sum_{j=1}^{m} X_{j}^{2} u$.

While it is relatively easy to see that, in any domain, weak solutions to this equation are bounded, further regularity is based on intricate subelliptic estimates. In Section 4 we show that any weak solution of the equation

$$
\mathscr{L}_{p} u=\sum_{j=1}^{n} X_{j}\left(|X u|^{p-2} X_{j} u\right)=-|u|^{p^{*}-2} u \quad \text { in } \boldsymbol{G} .
$$

is a bounded function. We shall present the proof in a somewhat more general case. There are several similar results in the Riemannian case. Yamabe [1960], proved the boundedness for solutions of the Yamabe problem on a manifold without boundary; see also [Trudinger 1968]. For the ordinary Laplacian 
in a bounded domain, Brézis and Kato [1979] established a result similar to part (1) of Theorem 4.1; for part (2), see [Brézis and Nirenberg 1983]. The proofs rely on a suitable modification of the test-function and truncation ideas introduced in Serrin's seminal paper [1964] and in [Moser 1961]. We note that, in all these results, $u$ is assumed from the space $L^{p}(\Omega)$, since this is part of the definition of the considered Sobolev spaces. This is not true in the Sobolev spaces $\mathscr{D}^{1, p}(\Omega)$ that we consider, since we only include the $L^{p}$ norm of the horizontal gradient in our definition. Therefore, the results here are not exactly the same, besides our working on a Carnot group. Subsequently, Serrin's ideas were generalized to the subelliptic setting in [Capogna et al. 1993] and, in different forms, also in [Holopainen 1992; Holopainen and Rickman 1992; Lanconelli and Uguzzoni 1998; Xu 1990].

The rest of the paper concerns the regularity of solutions on bounded domains. In Section 5 we show that, under certain geometric conditions on the boundary of the $C^{\infty}$ connected bounded open set $\Omega \subset \boldsymbol{G}$, one can prove boundedness of the horizontal gradient of the Green's function. We shall also obtain estimates along other vector fields. Similar results hold near the boundary of the domain for weak nonnegative solutions of the following Yamabe-type equation

$$
\mathscr{L} u=-u^{2^{*}-1}, \quad u \in \mathscr{D}^{1,2}(\Omega), \quad u \geq 0 .
$$

In order to prove such estimates, we impose some geometrical conditions on the boundary. In particular, we point to the "convexity condition" [Garofalo and Vassilev 2000], assumed to hold globally whenever we are working with nonlinear equations, unless we are on the Heisenberg group. The reason for such an assumption is that, at present, there is no proof of the boundary Schauder estimates in Lipschitz spaces, or of $\Gamma^{2, \alpha}$ regularity near the points from the noncharacteristic portion of the boundary. Assuming the global validity of the convexity condition allows us to avoid the use of Schauder estimates or extra a priori regularity assumptions. However, by using the Lipschitz Schauder theory near the noncharacteristic boundary for domains on the Heisenberg group [Jerison 1981a], we present in Section 5E the argument in the local setting, with the convexity assumption holding only near the characteristic boundary.

An example in [Jerison 1981b] implies that the estimates we obtain at the characteristic boundary fail without some assumption on the boundary. However, the convexity condition is clearly not necessary for estimates of the type considered. Boundedness of the horizontal gradient has been established for more general domains, satisfying a uniform outer-ball condition [Capogna et al. 1998]. Verifying such a condition is in general hard. A result as in the $C^{1,1}$-boundary case in $\mathbb{R}^{n}$ is not true. Also, the horizontal gradient involves only differentiation along vectors from the first layer. We give estimates along vector fields involving differentiation along vectors from other layers; in particular, we include the radial vector field. 
Lastly, in [Garofalo and Vassilev 2000] it was required that the domain be uniformly star-like near the characteristic boundary, which is unnecessary, as we will show.

\section{Preliminaries and notation}

We introduce the relevant definitions and state some results which will be needed in the sequel. Consider the Lie algebra $\mathfrak{g}=\bigoplus_{j=1}^{r} V_{j}$ of $\boldsymbol{G}$. We assume that there is a scalar product on $\mathfrak{g}$, with respect to which the $V_{j}$ 's are mutually orthogonal.

Let $X=\left\{X_{1}, \ldots, X_{m}\right\}$ be a basis of $V_{1}$, and continue to denote by $X$ the corresponding system of sections on $\boldsymbol{G}$. The sub-Laplacian associated with $X$ is the second-order partial differential operator on $\boldsymbol{G}$ given by

$$
\mathscr{L}=-\sum_{j=1}^{m} X_{j}^{*} X_{j}=\sum_{j=1}^{m} X_{j}^{2}
$$

(recall that $X_{j}^{*}=-X_{j}$ in a Carnot group; see [Folland 1975]). From the assumption on the Lie algebra, one immediately sees that the system $X$ satisfies the well-known finite-rank condition, and therefore that the operator $\mathscr{L}$ is hypoelliptic, thanks to Hörmander's theorem [1967]. However, it fails to be elliptic, and the loss of regularity is measured by the step $r$ of the stratification of $\mathfrak{g}$. For a function $u$ on $\boldsymbol{G}$, set

$$
|X u|=\left(\sum_{j=1}^{m}\left(X_{j} u\right)^{2}\right)^{1 / 2} .
$$

For $1 \leq p<Q$, define $\grave{D}^{1, p}(\boldsymbol{G})$ as the closure of $C_{0}^{\infty}(\boldsymbol{G})$ with respect to the norm

$$
\|u\|_{\mathfrak{D}^{1} 1, p(\boldsymbol{G})}=\left(\int_{\boldsymbol{G}}|X u|^{p} d H\right)^{1 / p} .
$$

We define the Sobolev exponent relative to $p$ as the number $p^{*}=p Q /(Q-p)$, where $Q$ is the homogeneous dimension defined below. The relevance of such a number is emphasized by the Folland-Stein inequality (1-1).

In any Carnot group, the exponential mapping exp : $\mathfrak{g} \rightarrow \boldsymbol{G}$ is an analytic diffeomorphism. We use it to define analytic maps $\xi_{i}: \boldsymbol{G} \rightarrow V_{i}, i=1, \ldots, r$, through the equation $g=\exp \xi(g)$, where $\xi(g)=\xi_{1}(g)+\cdots+\xi_{r}(g)$. With $m=\operatorname{dim}\left(V_{1}\right)$, the coordinates of $\xi$ 's projection in the basis $X_{1}, \ldots, X_{m}$ will be denoted by $x_{1}=x_{1}(g)$, $\ldots, x_{m}=x_{m}(g)$, that is,

$$
x_{j}(g)=\left\langle\xi(g), X_{j}\right\rangle, \quad j=1, \ldots, m .
$$

We set $x=x(g)=\left(x_{1}, \ldots, x_{m}\right) \in \mathbb{R}^{m}$. Later we will need to exploit the properties of the exponential coordinates in the second layer of the stratification of $\mathfrak{g}$. We 
thus fix an orthonormal basis $Y_{1}, \ldots, Y_{k}$ of $V_{2}$ and, similarly to (2-2), define the exponential coordinates in the second layer $V_{2}$ of a point $g \in \boldsymbol{G}$ by setting

$$
y_{i}(g)=\left\langle\xi(g), Y_{i}\right\rangle, \quad i=1 \ldots k,
$$

and $y=\left(y_{1}, \ldots, y_{k}\right) \in \mathbb{R}^{k}$.

Every Carnot group is naturally equipped with a family of nonisotropic dilations defined by

$$
\delta_{\lambda}(g)=\exp \circ \Delta_{\lambda} \circ \exp ^{-1}(g), \quad g \in \boldsymbol{G},
$$

where $\exp : \mathfrak{g} \rightarrow \boldsymbol{G}$ is the exponential map and $\Delta_{\lambda}: \mathfrak{g} \rightarrow \mathfrak{g}$ is defined by

$$
\Delta_{\lambda}\left(\xi_{1}+\cdots+\xi_{r}\right)=\lambda \xi_{1}+\cdots+\lambda^{r} \xi_{r} .
$$

The topological dimension of $\boldsymbol{G}$ is $N=\sum_{j=1}^{r} \operatorname{dim} V_{j}$, whereas the homogeneous dimension of $\boldsymbol{G}$, attached to the automorphisms $\left\{\delta_{\lambda}\right\}_{\lambda>0}$, is given by

$$
Q=\sum_{j=1}^{r} j \operatorname{dim} V_{j} .
$$

One has $d H\left(\delta_{\lambda}(g)\right)=\lambda^{Q} d H(g)$, so that, with respect to the group dilations, the number $Q$ plays the role of a dimension. Let $Z$ be the infinitesimal generator of the one-parameter group of nonisotropic dilations $\left\{\delta_{\lambda}\right\}_{\lambda>0}$. Such a vector field is characterized by the property that a function $u: G \rightarrow \mathbb{R}$ is homogeneous of degree $s$ with respect to $\left\{\delta_{\lambda}\right\}_{\lambda>0}$ - that is, $u\left(\delta_{\lambda}(x)\right)=\lambda^{s} u(x)$ for every $x \in \boldsymbol{G}$ - if and only if $Z u=s u$.

The Euclidean distance to the origin $|\cdot|$ on $\mathfrak{g}$ induces a homogeneous norm $|\cdot|_{\mathfrak{g}}$ on $\mathfrak{g}$, and (via the exponential map) a norm on the group $\boldsymbol{G}$, in the following way (see also [Folland 1975]): First, for $\xi \in \mathfrak{g}$ with $\xi=\xi_{1}+\cdots+\xi_{r}, \xi_{i} \in V_{i}$, set

$$
|\xi|_{\mathfrak{g}}=\left(\sum_{i=1}^{r}\left|\xi_{i}\right|^{2 r ! / i}\right)^{2 r !}
$$

then define $|g|_{\boldsymbol{G}}=|\xi|_{\mathfrak{g}}$ if $g=\exp \xi$. Such a norm on $\boldsymbol{G}$ can be used to define a pseudodistance on $\boldsymbol{G}$ :

$$
\rho(g, h)=\left|h^{-1} g\right|_{G} .
$$

The pseudodistance $\rho$ is equivalent to the Carnot-Carathéodory distance $d(\cdot, \cdot)$ generated by the system $X$, that is, there exists a constant $C=C(\boldsymbol{G})>0$ such that

$$
C \rho(g, h) \leq d(g, h) \leq C^{-1} \rho(g, h), \quad g, h \in \boldsymbol{G},
$$

see [Nagel et al. 1985]. We will almost exclusively work with the distance $d$, except in a few situations where we will find more convenient to use (2-6). The 
Carnot-Carathéodory balls are defined in the obvious way,

$$
B_{R}(x) \equiv B(x, R)=\{y \in \boldsymbol{G} \mid d(x, y)<R\} .
$$

By left-translation and dilation it is easy to see that the Haar measure of $B(x, R)$ is proportional to $R^{Q}$, where $Q=\sum_{i=1}^{r} i \operatorname{dim} V_{i}$ is the homogeneous dimension of $\boldsymbol{G}$. One has, for every $f, g, h \in \boldsymbol{G}$ and any $\lambda>0$,

$$
d(g f, g h)=d(f, h), \quad d\left(\delta_{\lambda}(g), \delta_{\lambda}(h)\right)=\lambda d(g, h) .
$$

We also recall the Baker-Campbell-Hausdorff formula; see, for example, [Hörmander 1967]:

$$
\exp \xi \exp \eta=\exp \left(\xi+\eta+\frac{1}{2}[\xi, \eta]+\cdots\right), \quad \xi, \eta \in \mathfrak{g},
$$

where the dots indicate a linear combination of terms of order three and higher, which is finite due to the nilpotency of $\boldsymbol{G}$. By definition, the order of an element in $V_{j}$ is $j$.

We next list some results which play an important role in this paper. To state these, we recall that, given a bounded open set $D \subset \boldsymbol{G}$ and a function $\varphi \in C(\partial D)$, the Dirichlet problem for $D$ and a sub-Laplacian $\mathscr{L}$ consists in finding a solution to $\mathscr{L} w=0$ in $D$ which takes value $\varphi$ on the boundary.

Theorem 2.1 (Bony's maximum principle [Bony 1969]). Let $D \subset \boldsymbol{G}$ be a connected bounded open set, and $\varphi \in C(\partial D)$. There exists a unique L-harmonic function $H_{\varphi}^{D}$ that solves the Dirichlet problem in the sense of Perron-Wiener-Brelot. Moreover, $H_{\varphi}^{D}$ satisfies

$$
\sup _{D}\left|H_{\varphi}^{D}\right| \leq \sup _{\partial D}|\varphi| .
$$

Theorem 2.2 (Schauder-type interior estimates [Danielli and Garofalo 1998]). Let $D \subset \boldsymbol{G}$ be an open set, and suppose that $w$ is $\mathscr{L}$-harmonic in $D$. For every $g \in D$ and $r>0$ with $\bar{B}(g, r) \subset D$, one has

$$
\left|X_{j_{1}} X_{j_{2}} \ldots X_{j_{s}} w(g)\right| \leq \frac{C}{r^{s}} \max _{\bar{B}(g, r)}|w|,
$$

for $s \in \mathbb{N}, j_{1}, \ldots, j_{s} \in\{1, \ldots, m\}$, and some constant $C=C(\boldsymbol{G}, s)>0$.

To state the next result, we introduce a definition. Given an open set $D \subset \boldsymbol{G}$, we denote by $\mathscr{L}^{1, \infty}(D)$ the space of those distributions $u \in L^{\infty}(D)$ such that $X u \in$ $L^{\infty}(D)$, endowed with the natural norm.

Theorem 2.3 ( $L^{\infty}$ Poincaré inequality [Garofalo and Nhieu 1998]). For a Carnot group $\boldsymbol{G}$, there exists $C=C(\boldsymbol{G})>0$ such that, if $u \in \mathscr{L}^{1, \infty}\left(B\left(g_{0}, 3 R\right)\right)$, then $u$ can be modified on a set of measure zero in $\bar{B}\left(g_{0}, R\right)$ so as to satisfy

$$
|u(g)-u(h)| \leq C d(g, h)\|u\|_{\mathscr{L}^{1, \infty}\left(B\left(g_{0}, 3 R\right)\right)}
$$


for every $g, h \in \bar{B}\left(g_{0}, R\right)$. If furthermore $u \in C^{\infty}\left(B\left(g_{0}, 3 R\right)\right)$, then only the $L^{\infty}$ norm of $X u$ suffices in the right-hand side of the previous inequality.

We note explicitly that the theorem asserts that every function $u \in \mathscr{L}^{1, \infty}\left(B\left(g_{0}, 3 R\right)\right)$ has a representative which is Lipschitz-continuous in $B\left(g_{0}, R\right)$ with respect to the Carnot-Carathéodory distance $d$. The reverse implication also holds; see [Garofalo and Nhieu 1998].

Finally, we recall a characterization of the nonisotropic Lipschitz spaces on Carnot groups [Krantz 1982]:

Theorem 2.4. $f \in \Gamma_{\alpha}(\boldsymbol{G})$ if and only if $f\left(g_{t}\right) \in \Lambda_{\alpha}$ for every horizontal curve $g_{t}$.

Here $\Gamma_{\alpha}(\boldsymbol{G})$ and $\Lambda_{\alpha}$ denote, correspondingly, the nonisotropic Lipschitz space of Folland and Stein on $\mathbb{R}^{N}$, and the isotropic Lipschitz space on $\mathbb{R}$. A curve is called horizontal if $\frac{d}{d t} g_{t} \in \operatorname{span}\left\{X_{1}, \ldots, X_{m}\right\}$.

\section{Variational problems}

We apply the concentration-compactness principle of Lions [1985a; 1985b] in the homogeneous setting of a Carnot group $\boldsymbol{G}$, to prove that, for any $1<p<Q$, the best constant in the Folland-Stein embedding (1-1) is achieved. A simple argument shows that, without any loss of generality, we can consider only nonnegative functions; we shall do that throughout this section. Consequently, we show that, for any such $p$, the quasilinear equation with critical exponent,

$$
\mathscr{L}_{p} u=\sum_{j=1}^{n} X_{j}\left(|X u|^{p-2} X_{j} u\right)=-u^{p^{*}-1} \quad \text { in } \boldsymbol{G},
$$

possesses a weak nonnegative solution that is also, up to a constant, an extremal for the following variational problem

$$
I \stackrel{\text { def }}{=} \inf \left\{\left.\int_{\boldsymbol{G}}|X u|^{p}\left|u \in C_{0}^{\infty}(\boldsymbol{G}), \int_{\boldsymbol{G}}\right| u\right|^{p^{*}}=1\right\} .
$$

We used that $\mathscr{L}_{p}(c u)=c^{p-1} \mathscr{L}_{p} u$ to reduce the equation given by the EulerLagrange multiplier to (3-1). We shall consider a similar problem when restricting the test functions to those having with a certain symmetry. We prove that the corresponding infimum is achieved again. The purpose of this section is to record such basic results.

It is still an open question to find the norm of the Folland-Stein embedding. An interesting and more accessible problem is obtained by requiring that the group be of Heisenberg type. The precise value of the norm of the embedding when considering only functions with symmetries and $p=2$ was found in [Garofalo and Vassilev 2001]. The proof required the existence result of this section. In 
the particular case of the Heisenberg group, this problem was solved without the symmetry restriction by Jerison and Lee [1988].

3A. The best constant in the Folland-Stein inequality. A minimizing sequence $\left\{u_{n}\right\} \in C_{0}^{\infty}(\boldsymbol{G})$ of the variational problem (3-2) is characterized by the properties

$$
\int_{\boldsymbol{G}}\left|u_{n}\right|^{p^{*}}=1 \quad \text { and } \quad \int_{\boldsymbol{G}}\left|X u_{n}\right|^{p} \underset{n \rightarrow \infty}{\longrightarrow} I .
$$

Two crucial aspects of equation (3-1) and of the above variational problem are their invariance with respect to group translations and dilations. The former is obvious, since the vector fields $X_{j}$ are left-invariant. The latter must be suitably interpreted, and follows from the observation that

$$
\mathscr{L}_{p}\left(u \circ \delta_{\lambda}\right)=\lambda^{p} \delta_{\lambda} \circ \mathscr{L}_{p} u .
$$

If we thus define, for a solution $u$ of (3-1) and for $\lambda>0$, the rescaled function $u_{\lambda}=\lambda^{\alpha} u \circ \delta_{\lambda}$, then it is clear that $u_{\lambda}$ satisfies (3-1) if and only if $\alpha=Q / p^{*}=$ $(Q-p) / p$. These considerations lead to the introduction, for $u \in C_{0}^{\infty}(\boldsymbol{G})$, of two new functions:

$$
\tau_{h} u \stackrel{\text { def }}{=} u \circ \tau_{h}, \quad h \in \boldsymbol{G},
$$

where $\tau_{h}: \boldsymbol{G} \rightarrow \boldsymbol{G}$ is the left-translation operator $\tau_{h}(g)=h g$; and

$$
u_{\lambda} \stackrel{\text { def }}{=} \lambda Q / p^{*} u \circ \delta_{\lambda}, \quad \lambda>0 .
$$

It is easy to see that the norms in the Folland-Stein inequality and the functionals in the variational problem (3-2) are invariant under the transformations (3-5) and (3-6). Only the second part requires a small computation, since $d H$ is bi-invariant under translations.

$$
\left\|\delta_{\lambda} u\right\|_{L^{p^{*}(G)}}^{p^{*}}=\int_{\boldsymbol{G}}\left|u\left(\delta_{\lambda} g\right)\right|^{p^{*}} d H(g)=\int_{\boldsymbol{G}}|u(g)|^{p^{*}} \lambda^{-Q} d H(g)=\lambda^{-Q}\|u\|_{L^{p^{*}(G)}}^{p^{*}} .
$$

This shows that $\left\|u_{\lambda}\right\|_{L^{p^{*}(G)}}=\|u\|_{L^{p^{*}(G)}}$. Similarly,

$$
\left\|\delta_{\lambda} u\right\|_{\mathscr{D} 1, p(\boldsymbol{G})}^{p}=\left\|\lambda \delta_{\lambda} X u\right\|_{L^{p^{*}(\boldsymbol{G})}}^{p}=\lambda^{p}\left\|\delta_{\lambda} X u\right\|_{L^{p^{*}(\boldsymbol{G})}}^{p}=\lambda^{p-Q}\|u\|_{\mathscr{D} 1, p(\boldsymbol{G})}^{p} .
$$

Taking into account that $p^{*}=p Q /(Q-p)$, we obtain $\left\|X u_{\lambda}\right\|_{\mathscr{D}^{1} 1, p(\boldsymbol{G})}=\|X u\|_{\mathscr{D}^{1, p}(\boldsymbol{G})}$.

The main result about the existence of global minimizers is this:

Theorem 3.1. Let $\boldsymbol{G}$ be a Carnot group, and consider the minimization problem (3-2). Every minimizing sequence $\left\{u_{n}\right\}$ of (3-2) is relatively compact in $\grave{D}^{1, p}(\boldsymbol{G})$, possibly after translating and dilating each of its elements using (3-5) and (3-6). In particular, there exists a minimum of (3-2), and the equation

$$
\mathscr{L}_{p} u=-u^{p^{*}-1}
$$


admits a nontrivial nonnegative solution $u \in \mathscr{D}^{1, p}(\boldsymbol{G})$.

The proof of Theorem 3.1 is based on an adaptation of the concentration-compactness principle. In such an adaptation, the Euclidean space $\mathbb{R}^{n}$ is replaced by a Carnot group $\boldsymbol{G}$, with its homogeneous structure and Carnot-Carathéodory distance. We mention that the implementation of Lions' program relies, among others, on the Rellich-Kondrachov compact embedding. In the subelliptic setting, the proof of this result requires a substantial amount of work. A general version of it was proved in [Garofalo and Nhieu 1996]; it states that, if $\Omega$ denotes a bounded $X$-PS domain (Poincaré-Sobolev domain) in a Carnot-Carathéodory space, then the embedding

$$
\mathscr{L}^{1, p}(\Omega) \subset L^{q}(\Omega)
$$

is compact provided that $1 \leq q<p^{*}=p Q /(Q-p)$. Here, $\mathscr{L}^{1, p}(\Omega)$ indicates the Sobolev space of those functions $f \in L^{p}(\Omega)$ such that $X f \in L^{p}(\Omega)$, endowed with the natural norm. Carnot groups are the basic models of Carnot-Carathéodory spaces. We shall apply such a result to an increasing sequence of bounded domains $\Omega_{k} \subset \Omega_{k+1} \subset \boldsymbol{G}$ such that $\Omega_{k} \nearrow \boldsymbol{G}$. As $\Omega_{k}$, we can take the Carnot-Carathéodory ball $B_{k}$ centered at the identity $e \in \boldsymbol{G}$ with radius $k$, since it was proved in [Franchi et al. 1994; Garofalo and Nhieu 1996] that such sets are $X$-PS domains.

An important tool is the concentration function of a measure:

Definition 3.2. For a nonnegative measure $d v$ on $\boldsymbol{G}$, define the concentration function $Q$ on $[0, \infty)$ by

$$
Q(r) \stackrel{\text { def }}{=} \sup _{g \in \boldsymbol{G}}\left(\int_{B_{r}(g)} d v\right) .
$$

For a function $f$ on $\boldsymbol{G}$, we will call the concentration function of $f$ the concentration function of the measure $|f|^{p^{*}} d H$.

Similarly to Lions' works, the crucial ingredients in the solution of the variational problem are the next lemmata. For the proofs, we refer to [Lions 1984a; 1984b]. As already mentioned, we have a suitable version of the Rellich-Kondrachov compact embedding to replace the usual embedding used in the proof of the next lemma:

Lemma 3.3. Suppose $v_{n}$ is a sequence of probability measures on $\boldsymbol{G}$. There exists a subsequence, which we denote by $d v_{n}$, such that exactly one of the following three conditions holds:

(1) Compactness: There is a sequence $\left(g_{n}\right) \in \boldsymbol{G}$ such that, for every $\varepsilon>0$, there exists $R>0$ so that, for every $n$,

$$
\int_{B\left(g_{n}, R\right)} d v_{n} \geq 1-\varepsilon
$$


(2) Vanishing: For all $R>0$, we have

$$
\lim _{n \rightarrow \infty}\left(\sup _{g \in \boldsymbol{G}} \int_{B(g, R)} d v_{n}\right)=0 .
$$

(3) Dichotomy: There exists a $\lambda$ with $0<\lambda<1$, such that, for every $\varepsilon>0$, there exist $R>0$ and a sequence $\left(g_{n}\right)$ with the property: Given $R^{\prime}>R$, there exist nonnegative measures $v_{n}^{1}$ and $v_{n}^{2}$ for which

$$
\begin{aligned}
& 0 \leq v_{n}^{1}+v_{n}^{2} \leq v_{n}, \\
& \operatorname{supp} v_{n}^{1} \subset B\left(g_{n}, R\right) \quad \text { and } \quad \operatorname{supp} v_{n}^{2} \subset \boldsymbol{G} \backslash B\left(g_{n}, R^{\prime}\right), \\
& \left|\lambda-\int v_{n}^{1}\right|+\left|(1-\lambda)-\int v_{n}^{2}\right| \leq \varepsilon
\end{aligned}
$$

Remark 3.4. Using diagonal subsequences, we can also achieve

$$
\begin{aligned}
& \operatorname{supp} v_{n}^{1} \subset B_{R_{n}}\left(g_{n}\right) \quad \text { and } \quad \operatorname{supp} v_{n}^{2} \subset \boldsymbol{G} \backslash B_{2 R_{n}}\left(g_{n}\right), \\
& \lim _{n \rightarrow \infty}\left|\lambda-\int_{\boldsymbol{G}} v_{n}^{1}\right|+\left|(1-\lambda)-\int_{\boldsymbol{G}} v_{n}^{2}\right|=0 .
\end{aligned}
$$

Lemma 3.5. Suppose $u_{n} \rightarrow u$ weak-* in $\grave{D}^{1, p}(\boldsymbol{G}), \mu_{n}=\left|X u_{n}\right|^{p} d H \rightarrow \mu$, and $v_{n}=\left|u_{n}\right|^{p^{*}} d H \rightarrow v$ weak-* in measure, where $\mu$ and $v$ are bounded nonnegative measures on $\boldsymbol{G}$. There exist points $g_{j} \in \boldsymbol{G}$ and real numbers $d_{j} \geq 0$ and $e_{j} \geq 0$, at most countably many nonzero, such that

$$
\begin{aligned}
& v=|u|^{p^{*}}+\sum_{j} d_{j} \delta_{g_{j}} \\
& \mu \geq|X u|^{p} d H+\sum_{j} e_{j} \delta_{g_{j}} \\
& I d_{j}{ }^{p / p^{*}} \leq e_{j},
\end{aligned}
$$

where I is the constant from (3-2). In particular,

$$
\sum d_{j}^{p / p^{*}}<\infty
$$

Proof of Theorem 3.1. Since $p>1$, we can assume that $u_{n} \rightarrow u$ weak-* in $\grave{D}^{1, p}(\boldsymbol{G})$ for some $u \in \mathscr{D}^{1, p}(\boldsymbol{G})$, by regarding $\mathscr{D}^{1, p}(\boldsymbol{G})$ as the dual of $\mathscr{D}^{1, p^{\prime}}(\boldsymbol{G})$, where $p^{\prime}$ is the Hölder conjugate of $p$. From the Folland-Stein embedding theorem, this is also true for the weak-* convergence in $L^{p^{*}}(\boldsymbol{G})$. We can also assume the minimizing sequence is a.e. pointwise convergent on $\boldsymbol{G}$. This follows easily from Rellich's theorem, applied successively to an exhaustion of $\boldsymbol{G}$ by an increasing sequence of Carnot-Carathéodory balls. 
The sequential lower semicontinuity of the norms shows that

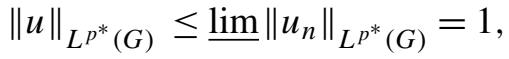

$$
\begin{aligned}
& \|u\|_{\mathfrak{D}^{1} 1, p(\boldsymbol{G})} \leq \underline{\lim }\left\|u_{n}\right\|_{\mathfrak{D}^{1} 1, p(\boldsymbol{G})}=I^{1 / p} .
\end{aligned}
$$

Thus, it is enough to prove that $\int_{G}|u|^{p^{*}}=1$, since, by the previous relations and the Folland-Stein inequality, and noticing that $I=1 / S_{p}^{p}$, we have

$$
I \geq \int_{\boldsymbol{G}}|X u|^{p} \geq I\left(\int_{\boldsymbol{G}}|u|^{p^{*}}\right)^{p / p^{*}}=I \quad \text { when } \int_{\boldsymbol{G}}|u|^{p^{*}}=1,
$$

which would give that $u$ is a minimizer. In other words, we reduce the proof to showing that $u_{n} \rightarrow u$ in $L^{p^{*}}(\boldsymbol{G})$, as weak-* and norm convergence imply strong convergence.

Because of translation and dilation invariance, all the mentioned properties hold if we replace $\left(u_{n}\right)$ with any translated and rescaled sequence, which we shall denote by $\left(v_{n}\right)$. We will consider the following measures,

$$
d v_{n} \stackrel{\text { def }}{=}\left|v_{n}\right|^{p^{*}} d H, \quad d \mu_{n} \stackrel{\text { def }}{=}\left|X v_{n}\right|^{p} d H .
$$

where $v_{n}$ is a suitable translation and dilation of $u_{n}$ that is to be defined in a moment. Note that $d v_{n}$ is also a sequence of probability measures. From the weak-* compactness of the unit ball, without loss of generality we can assume that $d v_{n} \rightarrow d v$ and $d \mu_{n} \rightarrow d \mu$ in the weak-* topology of all bounded nonnegative measures.

The desired convergence, that is, the fact that $\int_{G}|v|^{p^{*}}=1$, will be obtained by applying the concentration-compactness principle, exactly as in [Lions 1985a] (see also [Struwe 1990]). We shall see that $v$ is a probability measure, as well as that $d v=\int_{G}|v|^{p^{*}}$. Here $v$ is the limit of the sequence $\left(v_{n}\right)$ taken in various spaces, as we did for the sequence $\left(u_{n}\right)$.

Let $\hat{Q}_{n}(r)$ be the concentration function of $u_{n}$, that is,

$$
\hat{Q}_{n}(r) \stackrel{\text { def }}{=} \sup _{h \in \boldsymbol{G}}\left(\int_{B_{r}(h)}\left|u_{n}\right|^{p^{*}} d H\right) .
$$

Clearly, $\hat{Q}_{n}(0)=0, \lim _{r \rightarrow \infty} \hat{Q}_{n}(r)=1$, and $\hat{Q}_{n}$ is a continuous nondecreasing function. Therefore, for every $n$ we can find an $r_{n}>0$ such that

$$
\hat{Q}_{n}\left(\frac{1}{r_{n}}\right)=\frac{1}{2} .
$$

Since the integral in (3-10) is absolutely continuous, it defines a continuous function of $h$, which, as $u_{n} \in L^{p^{*}}(\boldsymbol{G})$, tends to zero when $d(h, e) \rightarrow \infty$. Consequently, 
the supremum is achieved, that is, for every $n$ there exist a $h_{n} \in \boldsymbol{G}$ such that

$$
\hat{Q}_{n}\left(\frac{1}{r_{n}}\right)=\int_{B_{1 / r_{n}}\left(h_{n}\right)}\left|u_{n}(g)\right| d H(g) .
$$

The concentration functions $Q_{n}$ of the dilated and translated sequence

$$
v_{n} \stackrel{\text { def }}{=} r_{n}^{-Q / p^{*}} \delta_{r_{n}^{-1}} \tau_{g_{n}} u
$$

satisfy

$$
Q_{n}(1)=\int_{B_{1}(e)} d v_{n} \quad \text { and } \quad Q_{n}(1)=\frac{1}{2},
$$

where we have set $g_{n}=\delta_{d_{n}} h_{n}^{-1}$.

The homogeneity properties of the metric are essential for proving (3-14). From the definition of $v_{n}$ and (2-8),

$$
\begin{aligned}
\int_{B_{r}(g)}\left|v_{n}(h)\right|^{p^{*}} d H(h) & =\int_{\{d(g, h)<r\}} r_{n}^{-Q}\left|u_{n}\left(\delta_{r_{n}^{-1}} \tau_{g_{n}} h\right)\right|^{p^{*}} d H(h) \\
& =\int_{\left\{d\left(g, \tau_{g_{n}-1} \delta_{r_{n}} z\right)<r\right\}} r_{n}^{-Q}\left|u_{n}(z)\right|^{p^{*}} r_{n}^{Q} d H(z) \\
& =\int_{\left\{d\left(\tau_{g_{n}} g, \delta_{r_{n}} z\right)<r\right\}}\left|u_{n}(z)\right|^{p^{*}} d H(z) \\
& =\int_{\left\{r_{m} d\left(\delta_{r_{n}-1} \tau_{g_{n}} g, z\right)<r\right\}}\left|u_{n}(z)\right|^{p^{*}} d H(z) \\
& =\int_{\left\{d(h, z)<r / r_{n}\right\}}\left|u_{n}(z)\right|^{p^{*}} d H(z),
\end{aligned}
$$

where $h=\delta_{r_{n}^{-1}} \tau_{g_{n}} g$. By taking the supremum over $g \in \boldsymbol{G}$, we obtain

$$
Q_{n}(1)=\hat{Q}_{n}\left(\frac{1}{r_{n}}\right)=\frac{1}{2} .
$$

When $g=e$, we have

$$
\int_{B_{1}(e)}\left|v_{n}(h)\right|^{p^{*}} d H(h)=\int_{B_{1 / r_{n}}\left(h_{n}\right)}\left|u_{n}(z)\right|^{p^{*}} d H(z),
$$

which shows that (3-14) holds.

At this point, we are ready to apply the lemmata and finish the proof. Notice that the vanishing case in Lemma 3.5 is ruled out by the normalization $Q_{n}(1)=1 / 2$. We embed our variational problem in the family

$$
I_{\lambda} \stackrel{\text { def }}{=} \inf \left\{\left.\int_{\boldsymbol{G}}|X u|^{p}\left|u \in C_{0}^{\infty}(\boldsymbol{G}), \int_{\boldsymbol{G}}\right| u\right|^{p^{*}}=\lambda\right\} .
$$


Since $I_{\lambda}=\lambda^{p / p^{*}} I$, we see that $I_{\lambda}$ is strictly subadditive, that is,

$$
I_{1}<I_{\alpha}+I_{1-\alpha} \quad \text { for every } \alpha \in(0,1) .
$$

Assume first that the compactness case in Lemma 3.3 holds when applied to the sequence $d v_{n}$. Let $g_{n}$ be points as in the lemma and, for $\varepsilon>0$, choose $R=R(\varepsilon)$, such that

$$
\int_{B_{R}\left(g_{n}\right)} d v_{n} \geq 1-\varepsilon \quad \text { for every } n
$$

If $\varepsilon<1 / 2$, then $\int_{B_{R}\left(g_{n}\right)} d v_{n}>1 / 2$. Since by construction $\int_{B_{1}(e)} d v_{n}=1 / 2$ while $\int_{G} d v_{n}=1$, we see that $B_{1}(e)$ and $B_{R}\left(g_{n}\right)$ have a nonempty intersection, and thus there exist a ball $B_{2 R+1}(e) \supset B_{R}\left(g_{n}\right)$. This implies that

$$
\int_{B_{2 R+1}(e)} d v_{n} \geq \int_{B_{R}\left(g_{n}\right)} d v_{n} \geq 1-\varepsilon \quad \text { for every } n,
$$

and therefore the condition (1) of Lemma 3.3 holds with $g_{n} \equiv e$ for every $n$. By taking $\varepsilon \rightarrow 0$, we proved that

$$
\int_{G} d v=1
$$

If we look at the sequence $\left(d \mu_{n}\right)$ now, we have $d \mu_{n} \rightarrow d \mu$ and $\int_{G} d \mu_{n} \rightarrow I$, and thus $\int_{G} d \mu \leq I$. On the other hand, Lemma 3.5 gives

$$
\begin{gathered}
d v_{n} \rightarrow d v=|v|^{p^{*}}+\sum_{j} d_{j} \delta_{g_{j}}, \\
d \mu_{n} \rightarrow d \mu \geq|X v|^{p} d H+\sum_{j} e_{j} \delta_{g_{j}},
\end{gathered}
$$

for certain $d_{j}, e_{j} \geq 0$ satisfying

$$
I d_{j}{ }^{p / p^{*}} \leq e_{j}
$$

We shall prove that all $d_{j}$ 's are zero, and thus $\int_{\boldsymbol{G}}|v|^{p^{*}} d H=1$. Let

$$
\alpha \stackrel{\text { def }}{=} \int_{G}|v|^{p^{*}} d H<1
$$

Since $\int_{\boldsymbol{G}} d \nu=1$, we have $\sum d_{j}=1-\alpha$. From $\int_{\boldsymbol{G}} d \mu \leq I$ we have

Now (3-23) gives

$$
\int_{G}|X v|^{p} d H \leq I-\sum e_{j}
$$

$$
I=I_{1} \geq \int_{G}|X v|^{p} d H+\sum e_{j} \geq I_{\alpha}+\sum I d_{j}^{p / p^{*}} \geq I_{\alpha}+\sum I_{d_{j}} .
$$


From the strict subadditivity (3-19) of $I_{\lambda}$, we conclude that exactly one of the numbers $\alpha$ and $d_{j}$ is nonzero. We claim that $\alpha=1$ (and thus that all $d_{j}$ 's are zero). Suppose that there is a $d_{j}$ with $d_{j}=1$ and $d v=\delta_{g_{j}}$. From the normalization, $Q_{n}(1)=1 / 2$, and hence

$$
\frac{1}{2} \geq \int_{B_{1}\left(g_{j}\right)}\left|v_{n}\right|^{p^{*}} d H \rightarrow \int_{B_{1}\left(g_{j}\right)} d v=1,
$$

which is a contradiction. Thus, we proved that $\|v\|_{L^{p^{*}}(G)}=\alpha=1$ and $v_{n} \rightarrow v$ in $L^{p^{*}}(\boldsymbol{G})$, which shows that $v$ is a solution of the variational problem; see (3-9).

Suppose we have dichotomy. We have to show that this leads to a contradiction. A simple process of taking a diagonal subsequence (see the remark after Lemma 3.3) shows that we can find a sequence $R_{n}>0$ such that

$$
\begin{gathered}
\operatorname{supp} v_{n}^{1} \subset B_{R_{n}}\left(g_{n}\right), \quad \operatorname{supp} v_{n}^{2} \subset \boldsymbol{G} \backslash B_{2 R_{n}}\left(g_{n}\right), \\
\lim _{n \rightarrow \infty}\left|\lambda-\int_{\boldsymbol{G}} v_{n}^{1}\right|+\left|(1-\lambda)-\int_{\boldsymbol{G}} v_{n}^{2}\right|=0 .
\end{gathered}
$$

We fix a number $\varepsilon$ such that

$$
0<\varepsilon<\lambda^{p / p^{*}}+(1-\lambda)^{p / p^{*}}-1 \text {. }
$$

Such a choice of $\varepsilon$ is possible, as for $0<\lambda<1$ and $p / p^{*}<1$ we have $\lambda^{p / p^{*}}+$ $(1-\lambda)^{p / p^{*}}-1>0$.

Let $\varphi$ be a cut-off function $0 \leq \varphi \in C_{0}^{\infty}\left(B_{2}(e)\right)$ with $\varphi \equiv 1$ on $B_{1}(e)$, and set $\varphi_{n}=\delta_{R_{n}^{-1}} \tau_{g_{n}} \varphi$. We have

$$
\int_{\boldsymbol{G}}\left|X v_{n}\right|^{p} d H=\int_{\boldsymbol{G}}\left|X\left(\varphi_{n} v_{n}\right)\right|^{p} d H+\int_{\boldsymbol{G}}\left|X\left(\left(1-\varphi_{n}\right) v_{n}\right)\right|^{p} d H+\varepsilon_{n} .
$$

Note that the remainder term $\varepsilon_{n}$ is expressed by an integral over an annulus

$$
A_{n}=B_{2 R_{n}}\left(g_{n}\right) \backslash B_{R_{n}}\left(g_{n}\right) .
$$

Furthermore, we claim that

$$
\varepsilon_{n} \geq o(1)-\varepsilon \int_{G}\left|X v_{n}\right|^{p} d H, \quad \text { where } o(1) \rightarrow 0 \text { as } n \rightarrow \infty .
$$

Indeed, using the inequality

$$
(|a|+|b|)^{p} \leq(1+\varepsilon)|a|^{p}+C_{\varepsilon, p}|b|^{p},
$$


which holds for any $0<\varepsilon<1, p \geq 1$, and a suitable constant $C_{\varepsilon, p}$ depending on $\varepsilon$ and $p$, we have

$$
\begin{aligned}
\varepsilon_{n} & =\int_{A_{n}}\left|X v_{n}\right|^{p} d H-\int_{A_{n}}\left|X\left(\varphi_{n} v_{n}\right)\right|^{p} d H-\int_{A_{n}}\left|X\left(\left(1-\varphi_{n}\right) v_{n}\right)\right|^{p} d H \\
& \geq \int_{A_{n}}\left|X v_{n}\right|^{p}\left(1-\varphi_{n}^{p}-\left(1-\varphi_{n}\right)^{p}\right) d H-2 C_{\varepsilon, p} \int_{A_{n}}\left|v_{n}\right|^{p}\left|X \varphi_{n}\right|^{p} d H \\
& -\varepsilon \int_{A_{n}}\left|X v_{n}\right|^{p} d H .
\end{aligned}
$$

Since $p>1$ and $0 \leq \varphi \leq 1$, it follows that $1 \geq \varphi_{n}{ }^{p}+\left(1-\varphi_{n}\right)^{p}$, and thus

$$
\varepsilon_{n} \geq-C \int_{A_{n}}\left|v_{n}\right|^{p}\left|X \varphi_{n}\right|^{p} d H-\varepsilon \int_{G}\left|X v_{n}\right|^{p} d H .
$$

First we use $\left|X \varphi_{n}\right| \leq C / R_{n}$, and then we apply Hölder's inequality on $A_{n}$,

$$
R_{n}^{-1}\left\|v_{n}\right\|_{L^{p}\left(A_{n}\right)} \leq R_{n}^{-1}\left|A_{n}\right|^{1 / p-1 / p^{*}}\left\|v_{n}\right\|_{L^{p^{*}}\left(A_{n}\right)} .
$$

Since $1 / p-1 / p^{*}=1 / Q$ and, from the paragraph above (2-8),

$$
\left|A_{n}\right| \sim R_{n}^{Q}
$$

we obtain

$$
R_{n}^{-1}\left\|v_{n}\right\|_{L^{p}\left(A_{n}\right)} \leq C\left\|v_{n}\right\|_{L^{p^{*}}\left(A_{n}\right)} .
$$

The last term in the above inequality can be estimated as follows:

$$
\begin{aligned}
\left\|v_{n}\right\|_{L^{p^{*}\left(A_{n}\right)}}^{p^{*}} & =\int_{A_{n}} d v_{n}=\int_{\boldsymbol{G}} d v_{n}-\int_{\boldsymbol{G} \backslash A_{n}} d v_{n} \\
& \leq \int_{\boldsymbol{G}} d v_{n}-\int_{\boldsymbol{G} \backslash A_{n}} d v_{n}^{1}-\int_{\boldsymbol{G} \backslash A_{n}} d v_{n}^{2} \\
& =\int_{\boldsymbol{G}} d v_{n}-\int_{\boldsymbol{G}} d v_{n}^{1}-\int_{\boldsymbol{G}} d v_{n}^{2} .
\end{aligned}
$$

Hence, the claim (3-30) follows from

(3-35) $R_{n}^{-1}\left\|v_{n}\right\|_{L^{p}\left(A_{n}\right)} \leq C\left(\int_{\boldsymbol{G}} d v_{n}-\int_{\boldsymbol{G}} d v_{n}^{1}-\int_{\boldsymbol{G}} d v_{n}^{2}\right)^{1 / p^{*}} \rightarrow 0 \quad$ as $n \rightarrow \infty$. 
Continuing with the proof that the dichotomy case does not occur, we use the definition of $I$, see (2-1) and (3-2), together with the above inequalities, to get

$$
\begin{aligned}
\left\|v_{n}\right\|_{\mathscr{\mathscr { D }} 1, p(\boldsymbol{G})}^{p} & =\left\|\varphi_{m} v_{n}\right\|_{\mathscr{\mathscr { D }} 1, p(\boldsymbol{G})}^{p}+\left\|\left(1-\varphi_{n}\right) v_{n}\right\|_{\mathscr{\mathscr { D }} 1, p(\boldsymbol{G})}^{p}+\varepsilon_{n} \\
& \geq I\left(\left\|\varphi_{m} v_{n}\right\|_{L^{p^{*}(G)}}^{p}+\left\|\left(1-\varphi_{n}\right) v_{n}\right\|_{L^{p^{*}(G)}}^{p}\right)+\varepsilon_{n} \\
& \geq I\left(\left(\int_{B_{R_{n}}\left(g_{n}\right)} d v_{n}\right)^{p / p^{*}}+\left(\int_{\boldsymbol{G} \backslash B_{R_{n}}\left(g_{n}\right)} d v_{n}\right)^{p / p^{*}}\right)+\varepsilon_{n} \\
& \geq I\left(\left(\int_{\boldsymbol{G}} d v_{n}^{1}\right)^{p / p^{*}}+\left(\int_{\boldsymbol{G}} d v_{n}^{2}\right)^{p / p^{*}}\right)+\varepsilon_{n} .
\end{aligned}
$$

Letting $n \rightarrow \infty$, we obtain

$$
I=\lim _{n \rightarrow \infty}\left\|v_{n}\right\|_{\mathscr{D} 1, p(\boldsymbol{G})}^{p} \geq I\left(\lambda^{p / p^{*}}+(1-\lambda)^{p / p^{*}}\right)-\varepsilon I,
$$

which is a contradiction with the choice of $\varepsilon$ in (3-27), and hence the dichotomy case of Lemma 3.3 cannot occur. The proof of the theorem is finished.

3B. The best constant in the presence of symmetries. We consider here the same problem as before, but we restrict the class of test functions.

Definition 3.6. Let $\boldsymbol{G}$ be a Carnot group with Lie algebra $\mathfrak{g}=V_{1} \oplus V_{2} \cdots \oplus V_{n}$. We say that a function $U: G \rightarrow \mathbb{R}$ has partial symmetry with respect to $g_{0}$ if there exist an element $g_{0} \in \boldsymbol{G}$ such that for every $g=\exp \left(\xi_{1}+\xi_{2}+\cdots+\xi_{n}\right) \in \boldsymbol{G}$ one has

$$
U\left(g_{0} g\right)=u\left(\left|\xi_{1}(g)\right|, \ldots,\left|\xi_{n-1}(g)\right|, \xi_{n}(g)\right),
$$

for some function $u:[0, \infty) \times \cdots \times[0, \infty) \times V_{n} \rightarrow \mathbb{R}$.

A function $U$ is said to have cylindrical symmetry if there exist $g_{0} \in \boldsymbol{G}$ and $\varphi:[0, \infty) \times \cdots \times[0, \infty) \rightarrow \mathbb{R}$ for which

$$
U\left(g_{0} g\right)=\varphi\left(\left|\xi_{1}(g)\right|,\left|\xi_{2}(g)\right|, \ldots,\left|\xi_{n}(g)\right|\right),
$$

for every $g \in \boldsymbol{G}$.

We also define the spaces $\grave{D}_{\mathrm{ps}}^{1, p}(\boldsymbol{G})$ and $\grave{D}_{\mathrm{cyl}}^{1, p}(\boldsymbol{G})$ by

$$
\begin{aligned}
& \mathscr{D}_{\mathrm{ps}}^{1, p}(\boldsymbol{G}) \stackrel{\text { def }}{=}\left\{u \in \mathscr{\mathscr { D }}^{1, p}(\Omega) \mid u(g)=u\left(\left|\xi_{1}(g)\right|, \ldots,\left|\xi_{n-1}(g)\right|, \xi_{n}(g)\right)\right\}, \\
& \check{\mathscr{D}}_{\text {cyl }}^{1, p}(\boldsymbol{G}) \stackrel{\text { def }}{=}\left\{u \in \mathscr{D}^{1, p}(\Omega) \mid u(g)=u\left(\left|\xi_{1}(g)\right|,\left|\xi_{2}(g)\right|, \ldots,\left|\xi_{n}(g)\right|\right)\right\} .
\end{aligned}
$$

The effect of the symmetries (see also [Lions 1985b]) is manifested in the fact that, if the limit measure given by Lemma 3.5 concentrates at a point, then it must concentrate on the whole orbit of the group of symmetries. Therefore, in the cylindrical case there could be no points of concentration except at the origin, 
while in the partially symmetric case the points of concentration lie in the center of the group.

Theorem 3.7. (1) The norm of the embedding $\stackrel{\mathscr{D}}{\mathrm{ps}}_{\mathrm{ps}}^{1, p}(\boldsymbol{G}) \subset L^{p^{*}}(\boldsymbol{G})$ is achieved.

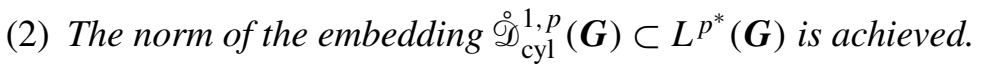

To rule out the dichotomy case in the first part of the theorem, we prove:

Lemma 3.8. Under the conditions of Lemma 3.3, the points $g_{n}$ in the dichotomy part can be taken from the center of the group.

Proof. Define the concentration function of $v_{n}$ by

$$
Q_{n}^{\mathrm{ps}} \stackrel{\text { def }}{=} \sup _{h \in C(\boldsymbol{G})}\left(\int_{B_{r}(h)} d v_{n}\right) .
$$

The rest of the proof is identical to the proof of Lemma 3.3, with the remark that, in the dichotomy part, the definition of $Q_{n}^{\mathrm{ps}}$ shows that the points $g_{n}$ can be taken to belong to the center.

Proof of Theorem 3.7. We argue as for Theorem 3.1.

(1) Finding the norm of the embedding $\stackrel{\mathscr{D}}{\mathrm{ps}}^{1, p}(\boldsymbol{G}) \subset \grave{D}^{1, p}(\Omega)$ leads to the variational problem

$$
I^{\mathrm{ps}} \equiv I_{1}^{\mathrm{ps}} \stackrel{\text { def }}{=} \inf \left(\int_{\boldsymbol{G}}|X u|^{p}: u \in \grave{D}_{\mathrm{ps}}^{1, p}(\boldsymbol{G}), \int_{\boldsymbol{G}}|u|^{p^{*}}=1\right) .
$$

We take a minimizing sequence $\left(u_{n}\right)$, that is,

$$
\int_{\boldsymbol{G}}\left|u_{n}\right|^{p^{*}}=1 \quad \text { and } \quad \int_{\boldsymbol{G}}\left|X u_{n}\right|^{p} \underset{n \rightarrow \infty}{\longrightarrow} I^{\mathrm{ps}} .
$$

It is clear that $\mathscr{D}_{\mathrm{ps}}^{1, p}(\boldsymbol{G})$ is invariant under the dilations (3-6). Using the BakerCampbell-Hausdorff formula, it is easy to see that $\mathscr{D}_{\mathrm{ps}}^{1, p}(\boldsymbol{G})$ is also invariant under the translations (3-5) by elements in the center $C(\boldsymbol{G})$ of $\boldsymbol{G}$. In order to extract a suitable dilated and translated subsequence of $\left\{u_{n}\right\}$, we have to make sure that we translate always by elements belonging to $C(\boldsymbol{G})$. For this, we define the concentration function of $u_{n}$ as

$$
\hat{Q}_{n}^{\mathrm{ps}}(r) \stackrel{\text { def }}{=} \sup _{h \in C(\boldsymbol{G})}\left(\int_{B_{r}(h)}\left|u_{n}\right|^{p^{*}} d H\right) .
$$

We can fix $r_{n}>0$ and $h_{n} \in C(\boldsymbol{G})$ such that (3-11) and (3-12) hold. Define the sequence $\left\{v_{n}\right\}$ as in (3-13). As mentioned above, $v_{n} \in \mathscr{D}_{\mathrm{ps}}^{1, p}(\boldsymbol{G})$ as well. Equation (3-15) holds without any changes. By taking the supremum over $g \in C(\boldsymbol{G})$ we obtain (3-16), and by using (3-17) we obtain (3-14). At this point, we apply Lemma 3.8. The case of vanishing is ruled out from the normalization (3-14) of 
the sequence $\left\{v_{n}\right\}$. Suppose we have dichotomy. As before, we take a sequence $R_{n}>0$ such that (3-25) and (3-26) hold. We choose a cut-off function $\varphi$ from the space $\stackrel{\circ}{\mathrm{ps}}_{\mathrm{ps}}^{1, p}(\boldsymbol{G})$, also satisfying

$$
\operatorname{supp} \varphi \subset \Omega_{2}(e) \quad \text { and } \quad \varphi \equiv 1 \quad \text { on } \Omega_{1}(e),
$$

where $\Omega_{r}(g)$ denotes a gauge ball centered at $g$ and of radius $r$, that is,

$$
\Omega_{r}(g)=\left\{h \in \boldsymbol{G} \mid N\left(h^{-1} g\right)<r\right\} .
$$

This can be done by setting $\varphi=\eta(N(g))$, where $\eta(t)$ is a smooth function on the real line, supported where $|t|<2$ and with $\eta \equiv 1$ on $|t| \leq 1$. We define the cut-off functions $\varphi_{n}$ as before, by $\varphi_{n}=\delta_{R_{n}^{-1}} \tau_{g_{n}} \varphi$. From Baker-Campbell-Hausdorff, they have partial symmetry with respect to the identity, since $g_{n} \in C(\boldsymbol{G})$ (see Lemma 3.8 ), and the gauge is a function with partial symmetry $\boldsymbol{G}$. By setting

$$
A_{n}=\Omega_{2 R_{n}}\left(g_{n}\right) \backslash \Omega_{R_{n}}\left(g_{n}\right)
$$

and noting that

$$
\left|A_{n}\right| \sim R_{n}^{Q}
$$

we see that (3-30) holds. Now, from the definition of $I_{\mathrm{ps}}$, keeping in mind that $\varphi_{n}$ and $v_{n}$ have partial symmetry with respect to the identity, we obtain

$$
\begin{aligned}
\left\|v_{n}\right\|_{\mathscr{\mathscr { D }} 1, p(\boldsymbol{G})}^{p} & =\left\|\varphi_{m} v_{n}\right\|_{\mathscr{\mathscr { D }} 1, p(\boldsymbol{G})}^{p}+\left\|\left(1-\varphi_{n}\right) v_{n}\right\|_{\mathscr{D} 1, p(\boldsymbol{G})}^{p}+\varepsilon_{n} \\
& \geq I_{\mathrm{ps}}\left(\left\|\varphi_{m} v_{n}\right\|_{L^{p^{*}(G)}}^{p}+\left\|\left(1-\varphi_{n}\right) v_{n}\right\|_{L^{p^{*}(G)}}^{p}\right)+\varepsilon_{n} \\
& \geq I_{\mathrm{ps}}\left(\left(\int_{B_{R_{n}}\left(g_{n}\right)} d v_{n}\right)^{p / p^{*}}+\left(\int_{\boldsymbol{G} \backslash B_{R_{n}}\left(g_{n}\right)} d v_{n}\right)^{p / p^{*}}\right)+\varepsilon_{n} \\
& \geq I_{\mathrm{ps}}\left(\left(\int_{\boldsymbol{G}} d v_{n}^{1}\right)^{p / p^{*}}+\left(\int_{\boldsymbol{G}} d v_{n}^{2}\right)^{p / p^{*}}\right)+\varepsilon_{n} \\
& \geq I_{\mathrm{ps}}\left(\lambda^{p / p^{*}}+(1-\lambda)^{p / p^{*}}\right)+\varepsilon_{n} .
\end{aligned}
$$

Letting $n \rightarrow \infty$, we come, as in (3-30), to

$$
\lim _{n \rightarrow \infty}\left\|v_{n}\right\|_{\mathscr{D}^{1} 1, p(\boldsymbol{G})}^{p} \geq I_{\mathrm{ps}}\left(\lambda^{p / p^{*}}+(1-\lambda)^{p / p^{*}}\right)>I_{\mathrm{ps}},
$$

since $0<\lambda<1$ and $p / p^{*}<1$. This contradicts that $\left\|v_{n}\right\|_{\mathscr{O} 1, p(\boldsymbol{G})}^{p} \rightarrow I^{\mathrm{ps}}$ as $n \rightarrow \infty$, which shows that the dichotomy case of Lemma 3.8 cannot occur. Hence, the compactness case holds. As in Theorem 3.1, we see that

$$
\int_{G} d v=1
$$


Next, we apply Lemma 3.5, with $I$ replaced by $I_{\mathrm{ps}}$. The important fact here is that the partial symmetry of the sequence $\left\{v_{n}\right\}$ implies that the points of concentration of $d \nu$, if they occur, must be in the center of the group. Having this in mind together with the definitions of the concentration functions, we can justify the validity of (3-24), and finish the proof of part (1).

(2) The vanishing case is ruled out by using the dilation (but not translation, because of the symmetries) invariance, and by normalizing the minimizing sequence with the condition $Q_{n}(1)=1 / 2$; see (3-14).

Suppose that the dichotomy case occurs. We shall see that this leads to a contradiction. The points $\left\{g_{n}\right\}$ in the dichotomy part of Lemma 3.3 must be a bounded sequence. If not, let $\varepsilon=\lambda / 2$ and $R$ as in the lemma. Because of the invariance under rotations in the layers of the functions $v_{n}$ and the Haar measure $d H$ (which is just the Lebesgue measure), for any arbitrarily fixed natural number $N_{0}$ we can find a point $g_{n}$ and $N_{0}$ points on the orbit of $g_{n}$ under rotations in one of the layers, such that the balls with radius $R$ centered at all these points do not intersect. This leads to a contradiction, since the integral of the probability measure $d v_{n}$ over each of these balls is greater than $\lambda / 2$. Thus, $\left\{g_{n}\right\}$ is a bounded sequence. This is, however, impossible since $d v_{n}$ are probability measures.

Therefore, the compactness case holds. As in the dichotomy part, we see that the sequence $\left\{g_{n}\right\} d v$ can concentrate only at the origin $e$. This is impossible.

\section{Global boundedness of weak solutions}

Let $p \in(1, Q)$ and denote by $p^{*}$ the Sobolev conjugate $p^{*}=p Q /(Q-p)$, and by $p^{\prime}$ the Hölder conjugate $p^{\prime}=p /(p-1)$. Let $u \in \mathscr{D}^{1, p}(\Omega)$ be a weak solution, not necessarily bounded, of the equation (1-3) in an open set $\Omega \subset \boldsymbol{G}$. "Weak solution" means that, for every $\varphi \in C_{0}^{\infty}(\Omega)$, we have

$$
\int_{\Omega}|X u|^{p-2}\langle X u, X \varphi\rangle d H=\int_{\Omega}|u|^{p^{*}-2} u \varphi d H .
$$

Note that $u^{p^{*}-1} \in L^{p^{*} /\left(p^{*}-1\right)}(\Omega)=L^{\left(p^{*}\right)^{\prime}}$. From the definition of $\mathscr{D}^{1, p}(\Omega)$, we obtain that (4-1) holds for every $\varphi \in \mathscr{D}^{1}, p(\Omega)$. The main result of this section is that weak solutions as above are bounded functions. In the next theorem we prove a more general result.

Theorem 4.1. Let $u \in \mathscr{D}^{1, p}(\Omega)$ be a weak solution to the equation

$$
\sum_{i=1}^{m} X_{i}\left(|X u|^{p-2} X_{i} u\right)=-V|u|^{p-2} u \quad \text { in } \Omega
$$


that is,

$$
\int_{\Omega}|X u|^{p-2}\langle X u, X \varphi\rangle d H=\int_{\Omega} V|u|^{p-2} u \varphi d H,
$$

for every $\varphi \in C_{0}^{\infty}(\Omega)$.

(1) If $V \in L^{Q / p}(\Omega)$, then $u \in L^{q}(\Omega)$ for every $p^{*} \leq q<\infty$.

(2) If $V \in L^{t}(\Omega) \cap L^{Q / p}(\Omega)$ for some $t>Q / p$, then $u \in L^{\infty}(\Omega)$.

Proof. The assumption that $V \in L^{Q / p}(\Omega)$, together with the Folland-Stein inequality, shows that (4-3) holds true for any $\varphi \in \mathscr{D}^{1, p}(\Omega)$. This can be seen by approximating in the space $\mathscr{D}^{1, p}(\Omega)$ by a sequence of test functions $\varphi_{n} \in C_{0}^{\infty}(\Omega)$, which will allow us to put the limit function in the left-hand side of (4-3). On the other hand, the Folland-Stein inequality implies that $\varphi_{n} \rightarrow \varphi$ in $L^{p^{*}}(\Omega)$. Set $t_{0}=Q / p$, and its Hölder conjugate $t_{0}^{\prime}=t_{0} /\left(t_{0}-1\right)$. An easy computation gives

$$
\frac{1}{t_{0}}+\frac{p-1}{p^{*}}=1-\frac{1}{p^{*}}=\frac{1}{\left(p^{*}\right)^{\prime}} .
$$

Hölder's inequality then shows that $V|u|^{p-2} u \in L^{\left(p^{*}\right)^{\prime}}(\Omega)$, which allows us to pass to the limit in the right-hand side of (4-3). We turn to the proofs of (1) and (2).

(1) It is enough to prove that, if $u \in \mathscr{D}^{1}, p(\Omega) \cap L^{q}(\Omega)$ with $q \geq p^{*}$, then $u \in L^{\kappa q}$ with $\kappa=p^{*} / p>1$. Let $G(t)$ be a continuous, piecewise-smooth, and globally Lipschitz function on the real line, and set

$$
F(u)=\int_{0}^{u}\left|G^{\prime}(t)\right|^{p} d t .
$$

Clearly, except at finitely many points, $F$ is a differentiable function with a bounded and continuous derivative. From the chain rule (see [Garofalo and Nhieu 1996], for example) there follows that $F(u) \in \mathscr{D}^{1, p}(\Omega)$ is a legitimate test function in (4-3). The left-hand side, taking into account that $F^{\prime}(u)=\left|G^{\prime}(u)\right|^{p}$, can be rewritten as

$$
\int_{\Omega}|X u|^{p-2}\langle X u, X F(u)\rangle d H=\int_{\Omega}|X G(u)|^{p} .
$$

The Folland-Stein inequality (1-1) gives

$$
\int_{\Omega}|X u|^{p-2}\langle X u, X F(u)\rangle d H \geq S_{p}\left(\int_{\Omega}|G(u)|^{p^{*}}\right)^{p / p^{*}} .
$$

We choose $G(t)$ as

$$
G(t)= \begin{cases}(\operatorname{sign} t)|t|^{q / p} & \text { if } 0 \leq|t| \leq l \\ l^{(q / p)-1} t & \text { if } l<|t|\end{cases}
$$


From the power growth of $G$, besides the preceding properties, this function also satisfies

$$
|u|^{p-1}|F(u)| \leq C(q)|G(u)|^{p} \leq C(q)|u|^{q} .
$$

The constant $C(q)$ depends also on $p$, but for us this is a fixed quantity. At this moment, the value of $C(q)$ is not important, but an easy calculation shows that $C(q) \leq C q^{p-1}$, with $C$ depending on $p$; we will use this in part (2). Note that $p t_{0}^{\prime}=p^{*}$. Leave $M>0$ to be fixed in a moment, and estimate the integral in the right-hand side of (4-3):

$$
\begin{array}{rl}
\int_{\Omega} V|u|^{p-2} u & F(u) d H \\
= & \int_{(|V| \leq M)} V|u|^{p-2} u F(u) d H+\int_{(|V|>M)} V|u|^{p-2} u F(u) d H \\
\leq & M \int_{(|V| \leq M)}|u|^{p-1} F(u) d H \\
& \quad+\left(\int_{(|V|>M)}|V|^{t_{0}} d H\right)^{1 / t_{0}}\left(\int_{\Omega}\left(|u|^{p-1} F(u)\right)^{t_{0}^{\prime}} d H\right)^{1 / t_{0}^{\prime}} \\
\leq C(q) M \int_{\Omega}|G(u)|^{p} d H & \quad C(q)\left(\int_{(|V|>M)}|V|^{t_{0}} d H\right)^{1 / t_{0}}\left(\int_{\Omega}|G(u)|^{p^{*}} d H\right)^{p / p^{*}} .
\end{array}
$$

At this point, we fix an $M$ sufficiently large so that

$$
C(q)\left(\int_{(|V|>M)}|V|^{t_{0}} d H\right)^{1 / t_{0}} \leq \frac{S_{p}}{2},
$$

which can be done because $V \in L^{t_{0}}$. Putting together (4-5) and (4-7) we come to our main inequality,

$$
\frac{S_{p}}{2}\left(\int_{\Omega}|G(u)|^{p^{*}} d H\right)^{p / p^{*}} \leq C(q) M \int_{\Omega}|G(u)|^{p} d H \leq C(q) M \int_{\Omega}|u|^{q} d H .
$$

By the Fatou and Lebesgue dominated convergence theorem, we can let $l$ in the definition of $G$ go to infinity, and obtain

$$
\frac{S_{p}}{2}\left(\int_{\Omega}|u|^{q p^{*} / p} d H\right)^{p / p^{*}} \leq C(q) M \int_{\Omega}|u|^{q} d H .
$$

The proof of (1) is finished. 
(2) It is enough to prove that the $L^{q}(\Omega)$ norms of $u$ are uniformly bounded by some sufficiently large but fixed $L^{q_{0}}$ norm of $u, q_{0} \geq p^{*}$, which is finite from (1). We shall do this by iteration. We use the function $F(u)$ from part (1) in the weak form (4-3) of our equation. The left-hand side is estimated from below as before, in (4-5). This time, though, we use Hölder's inequality to estimate from above the right-hand side:

$$
\begin{aligned}
\int_{\Omega} V|u|^{p-2} u F(u) d H & \leq\|V\|_{t}\left\||u|^{p-1} F(u)\right\|_{t^{\prime}} \\
& \leq\|V\|_{t}\left\|C(q)|G(u)|^{p}\right\|_{t^{\prime}} \leq C(q)\|V\|_{t}\|u\|_{q t^{\prime}}^{q} .
\end{aligned}
$$

With the estimate from below, we come to

$$
S_{p}\|G(u)\|_{p^{*}}^{p} \leq C(q)\|V\|_{t}\|u\|_{q t^{\prime}}^{q} .
$$

Letting $l \rightarrow \infty$, we obtain

$$
\left\||u|^{q / p}\right\|_{p^{*}}^{p} \leq \frac{C(q)}{S_{p}}\|V\|_{t}\|u\|_{q t^{\prime}}^{q} .
$$

Set $\delta=p^{*} /\left(p t^{\prime}\right)$. The assumption $t>Q / p$ implies $\delta>1$, since the latter is equivalent to $t^{\prime}<p^{*} / p=t_{0}^{\prime}$, as $t_{0}=Q / p$. With this notation we can rewrite (4-9) as

$$
\|u\|_{\delta q t^{\prime}} \leq\left(\frac{C(q)}{S_{p}}\right)^{1 / q}\|V\|_{t}^{1 / q}\|u\|_{q t^{\prime}} .
$$

Recall that $C(q) \leq C q^{p-1}$. At this point, we define $q_{0}=p^{*} t^{\prime}$ and $q_{k}=\delta^{k} q_{0}$. After a simple induction, we obtain

$$
\|u\|_{q_{k}} \leq \prod_{j=0}^{k-1}\left(C q_{j}^{p-1}\right)^{t^{\prime} / q_{j}}\|V\|_{t}^{t^{\prime} \sum_{j=0}^{k-1} 1 / q_{j}}\|u\|_{q_{0}} .
$$

We observe that the right-hand side is finite,

$$
\sum_{j=0}^{\infty} \frac{1}{q_{j}}=\frac{1}{q_{0}} \sum_{j=1}^{\infty} \frac{1}{\delta^{j}}<\infty \quad \text { and } \quad \sum_{j=1}^{\infty} \frac{\log q_{j}}{q_{j}}<\infty,
$$

because $\delta>1$. Letting $j \rightarrow \infty$, we obtain

$$
\|u\|_{\infty} \leq C\|u\|_{q_{0}} .
$$

Remark 4.2. When $\Omega$ is a bounded open set, we trivially have $V \in L^{Q / p}(\Omega)$ whenever $V \in L^{t}(\Omega)$ with $t>Q / p$. Also, in this case one can obtain a uniform estimate of the $L^{\infty}(\Omega)$ norm of $u$ by its $L^{p^{*}}(\Omega)$ norm that does not depend on the distribution function of $V$, as we had in the preceding theorem. This can even be 
achieved in the unbounded case, assuming only that $V \in L^{Q / p}(\Omega)$, but requiring $u \in L^{p}(\Omega)$.

With the previous theorem proved, we turn to our original equation (1-3):

Theorem 4.3. Take $p \in(1, Q)$ and let $\Omega \subset \boldsymbol{G}$ be an open set. If $u \in \mathscr{D}^{1, p}(\Omega)$ is a weak solution to the equation

$$
\mathscr{L}_{p} u=\sum_{j=1}^{n} X_{j}\left(|X u|^{p-2} X_{j} u\right)=-|u|^{p^{*}-2} u \quad \text { in } \Omega,
$$

then $u \in L^{\infty}(\Omega)$.

Proof. We define $V=|u|^{p^{*}-p}$. From the Folland-Stein inequality we have $u \in$ $L^{p^{*}}(\Omega)$, and thus $V \in L^{p^{*} /\left(p^{*}-p\right)}(\Omega)$. Since $p^{*} /\left(p^{*}-p\right)=Q / p$, part (1) of Theorem 4.1 shows that $u \in L^{q}(\Omega)$ for $p^{*} \leq q<\infty$. Therefore, $V \in L^{q /\left(p^{*}-p\right)}(\Omega)$ for any such $q$ and thus, by part (2) of the same theorem, we conclude that $u \in$ $L^{\infty}(\Omega)$.

\section{Regularity near the characteristic boundary}

We start by introducing the geometric assumptions on the domain, and describe the regularity of the weak solutions to the Yamabe equation (1-4), which can be obtained from well-known results.

Let $\Omega \subset \boldsymbol{G}$ be a $C^{\infty}$ domain whose boundary $\partial \Omega$ is an orientable hypersurface. We assume the existence of $\rho \in C^{\infty}(\boldsymbol{G})$ and $\gamma_{\Omega}>0$ such that, for some $R \in \mathbb{R}$,

$$
\Omega=\{g \in \boldsymbol{G} \mid \rho(g)<R\},
$$

and such that $|D \rho(g)| \geq \gamma_{\Omega}>0$ for every $g$ in some relatively compact neighborhood $K$ of $\partial \Omega$. We shall denote by $U$ a bounded open set such that $\partial \Omega \subset U$, and set $\omega=\Omega \cap U$. We stress that, with this assumption, $\partial \omega$ is a compact set inside $\Omega$. However, in Sections $5 \mathrm{~B}, 5 \mathrm{C}$, and $5 \mathrm{E}$ we shall make a different assumption, requiring that $U$ contain only the characteristic points of the boundary, and hence $\partial \omega$ will reach $\partial \Omega$.

The assumptions on the domains are:

- A-condition: There exist $A, r_{0}>0$ such that, for every $g \in \partial \Omega$ and $r \in\left(0, r_{0}\right)$,

$$
|(\boldsymbol{G} \backslash \Omega) \cap B(g, r)| \geq A|B(g, r)| .
$$

- Convexity: There exists $M_{1}>0$ such that the defining function $\rho$ of $\Omega$ satisfies the differential inequality

$$
\mathscr{L} \rho \geq \frac{2}{M_{1}}\langle X \rho, X \psi\rangle \quad \text { in } \omega,
$$


where $\psi(g) \stackrel{\text { def }}{=}|x(g)|^{2}$.

We remark that the $A$-condition is fulfilled if, for example, $\Omega$ satisfies the uniform corkscrew condition, see [Capogna and Garofalo 1998; Capogna et al. 1998]. These papers contain an extensive study of examples of domains that, in particular, satisfy (5-2). The $A$-condition allows us to adapt to the present setting Moser's [1961] classical iteration arguments. On the other hand, the convexity condition allows the construction of useful barriers. It is satisfied if, for example, $\rho$ is a strictly $\mathscr{L}$-superharmonic function.

From Theorem 4.3, we know that $u \in L^{\infty}(\Omega)$. This crucial information allows us to implement the local regularity theory of Folland and Stein [1974; Folland 1975 ] to conclude that $u \in C^{\infty}(\Omega)$.

The $A$-condition (5-2) permits us to adapt to the present setting the classical arguments that lead, via Moser's iteration, to $u \in \Gamma^{0, \alpha}(\bar{\Omega})$ for some $0<\alpha<1$; see for example [Gilbarg and Trudinger 1983, Section 8.10]. Extending $u$ by zero outside $\Omega$, we can assume henceforth that

$$
u \in \Gamma^{0, \alpha}(\boldsymbol{G}) \cap C^{\infty}(\Omega) .
$$

Given the domain $\Omega$ and an open neighborhood $U$, fixed as in the preceding discussion, we assume that $M_{0}>0$ has been chosen so that it fulfill the condition

$$
\bar{U} \subset\left\{\left.g \in \boldsymbol{G}|| x(g)\right|^{2} \leq m M_{0} / 4\right\} .
$$

We shall consider the Riemannian distance $d_{R}(\cdot, \cdot)$ on $\boldsymbol{G}$, defined using the Euclidean metric on $\mathfrak{g}$ via the exponential map; that is, if $g=\exp \xi$ and $h=\exp \eta$, we set

$$
d_{R}(g, h)=|\xi-\eta|
$$

It is straightforward to estimate the Riemannian distance by the Carnot-Carathéodory distance:

$$
d_{R}(g, h) \leq d(g, h) .
$$

The estimate in the other direction was proven by Tanaka [1975]. He works in a more general situation than ours, so we state the result as relevant to our setting.

Theorem 5.1. Let $\boldsymbol{G}$ be a Carnot group of step $r$, and take $g_{0} \in \boldsymbol{G}$. For every ball $B\left(g_{0}, R\right)$ there exists a constant $C=C(\boldsymbol{G}, R)$ such that, if $g, h \in B\left(g_{0}, R\right)$, then

$$
d(g, h) \leq C d_{R}(g, h)^{1 / r} .
$$

5A. Barrier functions. The barrier functions defined in the next theorem are crucial to the rest of the section. The convexity property of the boundary is essentially what makes these functions useful. 
Theorem 5.2. Let $\Omega \subset \boldsymbol{G}$ be a smooth connected bounded open set that satisfies the convexity condition (5-3). Let $M \geq \max \left\{M_{0}, M_{1}\right\}$. For $0<\alpha \leq 1$, we define

$$
\Psi_{\alpha}=(R-\rho)^{\alpha} e^{-\psi / M} .
$$

Under the stated hypothesis, for every $g \in \omega$,

$$
\mathscr{L} \Psi_{\alpha}(g) \leq-\frac{m}{M} \Psi_{\alpha}(g) .
$$

We remark that, when working with $\mathscr{L}$-harmonic functions, one takes $\alpha=1$. We also consider $\alpha<1$ because of its use in the case of nonlinear equations, for example, Yamabe-type equations. The proof of the above theorem can be found in [Garofalo and Vassilev 2000].

Let $\eta$ be a smooth vector field defined in $U$ that is transversal to the boundary, that is,

$$
\left.\eta \rho\left(g_{0}\right) \equiv\left(\frac{d}{d t} \rho\left(g_{t}\right)\right)\right|_{t=0} \neq 0, \quad g_{0} \in \partial \Omega,
$$

where $\frac{d}{d t} g_{t}=\eta\left(g_{t}\right)$.

Using the compactness of $\partial \Omega$, we can assume, possibly after taking a smaller $U$, that there exists a constant $\delta>0$ such that

$$
\eta \rho\left(g_{0}\right) \geq \delta>0 \quad \text { for } g_{0} \in \partial \Omega .
$$

We note that the transversality condition (5-11) implies that the trajectories of $\eta$ that start from points of $\partial \Omega$ fill a full open set $\omega$, interior to $\Omega$. Possibly by shrinking the set $U$, we can assume that $\omega=\Omega \cap U$. To fix the notation, we suppose that there exists $t_{0} \in(0,1)$ such that

$$
g_{t} \in \omega \quad \text { for } 0<t<t_{0} \text { and } g_{0} \in \partial \Omega .
$$

We shall hereafter use this generic transversal vector field and notation.

Lemma 5.3. There exist $C_{1}, C_{2}>0$ such that, for every $g_{0} \in \partial \Omega$ and $0 \leq t \leq t_{0}$,

$$
C_{1} t^{\alpha} \leq \Psi_{\alpha}\left(g_{t}\right) \leq C_{2} t^{\alpha} .
$$

Proof. Under the assumptions we made, the proof follows from the Taylor formula. Indeed,

$$
R-\rho\left(g_{t}\right)=\operatorname{t\eta } \rho\left(g_{0}\right)(1+O(t)),
$$

where $O(t)$ denotes a function bounded by $C t$, uniformly in $g_{0} \in \partial \Omega$ and $0 \leq t \leq t_{0}$.

It is clear from the latter identity that (5-12) follows by using (5-11), the smoothness of $\rho$, and the boundedness of $\Omega$. 
5B. Lipschitz estimates near the boundary. We start with a theorem about the Green's function, since its proof is very simple because of the existence of barriers.

An important observation is that, thanks to the results of Derridj [1971], the Green's function is a smooth function up to the noncharacteristic boundary; indeed, its $\mathscr{L}$-Laplacian vanishes near the boundary, and hence it is a smooth function. Therefore, when working with equations with a smooth right-hand side, we shall consider $\omega=U \cap \Omega$, where $U$ is a sufficiently small open neighborhood of the characteristic set $\Sigma$ defined in (5-37), rather than $\partial \Omega \subset U$ as we do in the case of the Yamabe equation; see the definitions of the convexity and $A$-conditions in Section 5A.

Theorem 5.4. Let $u \in C(\bar{\omega})$ satisfy

$$
\mathscr{L} u=0 \quad \text { in } \omega, \quad u=0 \quad \text { on } \partial \Omega .
$$

If the convexity condition is satisfied in a neighborhood $\omega$ of the characteristic boundary $\Sigma$, then there exists a constant $C=C(\boldsymbol{G}, \Omega, u)$ such that

$$
|u(g)| \leq C d_{R}(g, \partial \Omega) \quad \text { for every } g \in \omega .
$$

Remark 5.5. Note that in this theorem the right-hand side uses the Riemannian distance. This is important for the estimates on derivatives that involve vectors not only from the first layer. Clearly, the same inequality holds for the CarnotCarathéodory distance.

Proof. The proof uses $\Psi$ as a barrier, and Bony's maximum principle. As we saw before the statement of the theorem, we have

$$
u \in C^{\infty}(\bar{\omega} \backslash \Sigma) .
$$

Equation (5-9) with $\Psi=\Psi_{1}$ shows that

$$
\pm \mathscr{L} u(g)=0 \geq \mathscr{L} C \Psi(g), \quad g \in \omega .
$$

On the other hand, for a sufficiently large constant $C$, we have the estimate

$$
C \Psi(g) \geq|u(g)| \quad \text { on } \partial \omega .
$$

To see that (5-15) holds, we argue as follows. Its validity is clear on $\partial \Omega$ since both $u$ and $\Psi$ vanish there. On the other hand, the compact set $\partial \omega \cap \Omega$ is at a fixed distance away from the characteristic boundary, and the estimate (5-15) follows since $\Psi, u \in C^{\infty}(\partial \omega \cap \Omega)$ and from Lemma 5.3; see the proof of Theorem 5.14 for further details.

We can now apply Bony's maximum principle (Theorem 2.1) and conclude that the bound holds inside as well:

$$
|u(g)| \leq C \Psi(g), \quad g \in \omega .
$$


This implies that $u$ satisfies the Riemannian Lipschitz estimate

$$
|u(g)| \leq C d_{R}(g, \partial \Omega), \quad g \in \omega,
$$

which completes the proof.

The next theorem was proved in [Garofalo and Vassilev 2000] by also requiring that $\Omega$ be uniformly starlike along $\Sigma$. A minor modification shows that this is unnecessary. We shall use $g_{t}$ as in (5-10).

Theorem 5.6. Let $\Omega$ be a $C^{\infty}$ open bounded set in a Carnot group $\boldsymbol{G}$, and $u$ a solution of the Yamabe equation (1-4). If the convexity and A-conditions are satisfied, then there exists a constant $C=C(G, \Omega, u)$ such that

$$
u(g) \leq C d_{R}(g, \partial \Omega) \quad \text { for every } g \in \omega .
$$

Proof. We begin by observing that, thanks to (5-4) and $u=0$ on $\partial \Omega$, we have, for any $g_{0} \in \partial \Omega$,

$$
u\left(g_{t}\right) \leq C d\left(g_{t}, g_{0}\right)^{\alpha} .
$$

Theorem 5.1 now gives, for every $g_{0} \in \partial \Omega$ and $0 \leq t \leq t_{0}$,

$$
d\left(g_{t}, g_{0}\right) \leq C d_{R}\left(g_{t}, g_{0}\right)^{1 / r} \leq C t^{1 / r}
$$

for some constant $C=C(\Omega)>0$. Using (5-17) and (5-18), and setting $\alpha_{0}=\alpha / r$, we infer that

$$
u\left(g_{t}\right) \leq C t^{\alpha_{0}}
$$

for every $g_{0} \in \partial \Omega$ and $0 \leq t \leq t_{0}<1$.

We now let $\sigma=2^{*}-1=(Q+2) /(Q-2)$. In the sequel it will be important that $\sigma>1$. Since it is clear that (5-19) continues to hold if in the right-hand side we raise $t$ to any exponent smaller than $\alpha_{0}$, we assume in what follows that $\sigma \alpha_{0}<1$ and that there is some $n \in \mathbb{N}$ for which

$$
\sigma^{n} \alpha_{0}=1 \text {. }
$$

Next, we use the barriers constructed in Theorem 5.2. For any point $g_{t} \in \omega$ we have, from (1-4), (5-19), (5-12), and (5-9), that

$$
\begin{aligned}
-\mathscr{L} u\left(g_{t}\right) & =u\left(g_{t}\right)^{\sigma} \leq C t^{\sigma \alpha_{0}} \\
& \leq C C_{1}^{-1} \Psi_{\sigma \alpha_{0}}\left(g_{t}\right) \leq-C C_{1}^{-1} M m^{-1} \mathscr{L} \Psi_{\sigma \alpha_{0}}\left(g_{t}\right) \\
& =-\mathscr{L}\left(C^{*} \Psi_{\sigma \alpha_{0}}\right)\left(g_{t}\right) .
\end{aligned}
$$

Keeping in mind that, as $g_{0}$ varies in $\partial \Omega$ and $t$ in $\left(0, t_{0}\right)$, the point $g_{t}$ covers $\omega$, we have proved that

$$
\mathscr{L}\left(C^{*} \Psi_{\sigma \alpha_{0}}-u\right) \leq 0 \quad \text { in } \omega .
$$


At this point, we observe that (possibly using a constant larger than $C^{*}$ ) we also have the estimate

$$
C^{*} \Psi_{\sigma \alpha_{0}} \geq u \quad \text { on } \partial \omega \text {. }
$$

To see that (5-21) holds, we argue as follows. It is clear that (5-21) holds on $\partial \omega \cap \partial \Omega$, since both $u$ and $\Psi_{\sigma \alpha_{0}}$ vanish there. On the other hand, the compact set $\partial \omega \cap \Omega$ is at a fixed distance away from the boundary, and therefore, using that $u$ and $\Psi_{\sigma \alpha_{0}}$ are smooth there and $\Psi_{\sigma \alpha_{0}}$ does not vanish, one trivially obtains the estimate by using the maximum of $u$.

We can now apply to $\omega$ Bony's maximum principle from Theorem 2.1 to infer that a similar estimate also holds in $\omega$. From this result and from the right-hand side of (5-12), we conclude that, for every $g_{0} \in \omega$ and $0<t<t_{0}$,

$$
u\left(g_{t}\right) \leq C t^{\sigma \alpha_{0}},
$$

which shows that we have improved on (5-19). It is now clear that, by repeating the above arguments $n$ times, where $n$ is as in (5-20), we shall reach the desired conclusion (5-16).

\section{C. Estimates for the Green's function.}

Theorem 5.7. Let $u \in C^{\infty}(\bar{\omega} \backslash \partial \Omega) \cap C(\bar{\omega})$ satisfy

$$
\mathscr{L} u=0 \quad \text { in } \omega, \quad u=0 \quad \text { on } \partial \Omega .
$$

If the convexity condition (5-3) is satisfied, then

$$
X u \in L^{\infty}(\omega) .
$$

The proof of Theorem 5.7 is an immediate consequence of Theorem 5.4, with the help of the Schauder-type estimates proved in [Danielli and Garofalo 1998].

Proof. Fix an arbitrary $g \in \omega$. With $r=\operatorname{dist}(g, \partial \omega) / 2$, consider the ball $B(g, r) \subset$ $\bar{B}(g, r) \subset \omega$. Applying the interior Schauder estimates to the $\mathscr{L}$-harmonic function $u$, one has

$$
|X u(g)| \leq \frac{C}{r} \sup _{B(g, r)}|u|,
$$

for some constant $C=C(\boldsymbol{G})$. At this point, we invoke Theorem 5.4, which implies, for any $g^{\prime} \in B(g, r)$, the inequality

$$
\left|u\left(g^{\prime}\right)\right| \leq C^{\prime} d\left(g^{\prime}, \partial \Omega\right) \leq C^{\prime}\left(d\left(g^{\prime}, g\right)+d(g, \partial \Omega)\right) \leq 2 C^{\prime} r,
$$

with a constant $C^{\prime}=C^{\prime}(\boldsymbol{G}, \omega, u)$. Substitution in (5-23) finishes the proof. 
In the next theorems we show the boundedness of derivatives along some transversal to the boundary vector fields. We denote by $\Sigma$ the characteristic boundary, which is defined in (5-37). Also, as in Theorem 5.4, $\omega=U \cap \Omega$ where $U$ is an open neighborhood of $\Sigma$. We further set $\Delta=\partial \Omega \cap U$.

Theorem 5.8. Suppose $\eta$ is a vector field from the center of the Lie algebra of $\boldsymbol{G}$. Let $\tilde{\eta}$ be the corresponding left-invariant vector field. Assume further that $\Omega \subset \boldsymbol{G}$ satisfies the convexity condition (5-3) and $\tilde{\eta}$ is transversal to $\Sigma$. If u satisfies

$$
\mathscr{L} u=0 \quad \text { in } \omega, \quad u=0 \quad \text { on } \partial \Omega,
$$

then

$$
\tilde{\eta} u \in L^{\infty}(\omega) .
$$

Proof. For $t$ very close to 0 , we define $\omega_{t}=\left\{g \exp t \eta \mid 0<t<t_{0}, g \in \omega\right\} \cap \omega$ and $\partial \Delta_{t}=\left\{g_{0} \exp t \eta \mid g_{0} \in \Delta\right\}$, and consider the difference quotients

$$
\varphi_{t}=\frac{1}{t}\left(R_{\exp t \eta} u-u\right), \quad g \in \omega_{t},
$$

where $R_{h} u(g)=u(g h)$ is the right-translations operator. We claim that there exists a constant $C>0$ such that, for all $t$ sufficiently close to 0 and $g \in \omega_{t}$, one has

$$
\left|\varphi_{t}(g)\right| \leq C
$$

Suppose the claim (5-25) is true. Passing to the limit as $t \rightarrow 0$, we conclude that $|\tilde{\eta} u(g)| \leq C$ for every $g \in \omega$, thus establishing the theorem.

We turn to the proof of $(5-25)$. Note that

$$
\mathscr{L} \varphi_{t}(g)=0 .
$$

That is, each of the functions $\varphi_{t}$ is $\mathscr{L}$-harmonic when $u$ is harmonic. This follows since the considered $\mathscr{L}$-Laplacian is left invariant and, when $\eta$ is in the center of $\mathfrak{g}$, we have that $h=\exp t \eta$ is in the center of the group, and hence the right and left translations by $h$ coincide.

From Bony's maximum principle (Theorem 2.1), it is therefore enough to prove that, for some $t_{1}$ close to $0,(5-25)$ holds for $g \in \partial \omega_{t}$ and $t \in\left(0, t_{1}\right)$. Note that $\partial \omega_{t}=\Delta_{t} \cup(\partial \omega \cap \Omega)$. We analyze the two portions separately. Since any point $g \in \Delta_{t}$ can be written as $g=g_{0} \exp t \eta$ for some $g_{0} \in \Delta$, we have

$$
\varphi_{t}(g)=\frac{u\left(g_{0} \exp t \eta\right)-u\left(g_{0}\right)}{t} .
$$

We recall Theorem 5.4, which gives

$$
\left|u\left(g_{0} \exp t \eta\right)\right| \leq C d_{R}\left(g_{0} \exp t \eta, g_{0}\right) \leq C t,
$$


with a fixed constant $C$ when $g_{0}$ belongs to a fixed compact set. This estimate shows that

$$
\left|\varphi_{t}(g)\right| \leq C, \quad g \in \partial \Omega_{t} .
$$

Finally, the same inequality on $\partial \omega \cap \Omega$ follows from the $C^{\infty}$ regularity of $u$ in $\Omega$. In conclusion, we have proved the claim (5-25), and therefore the theorem.

Remark 5.9. The same proof can be used to show boundedness of the derivative along the radial vector field, assuming that it is transversal to $\partial \Omega$. The reason is that the corresponding difference quotients are $\mathscr{L}$-harmonic as well.

5D. Regularity of solutions of the Yamabe equation. Let $u$ be a weak nonnegative solution of the Yamabe-type equation (1-4). We note again that, because of the present lack of boundary Schauder estimates (except in the case of the Heisenberg group: see 5.13), in the next theorem the convexity assumption is required to hold globally. Accordingly, for domains in the Heisenberg group, convexity will be assumed only near the characteristic boundary; see Theorem 5.14.

Theorem 5.10. Let $\Omega$ be a $C^{\infty}$ open bounded set in a Carnot group $\boldsymbol{G}$, and $u$ a solution of (1-4). If the convexity assumption (5-3) and the A-condition (5-2) are satisfied, then

$$
X u \in L^{\infty}(\Omega) .
$$

Proof. Since $u$ is smooth away from $\partial \Omega$, in order to prove the theorem it will be enough to show that

$$
X u \in L^{\infty}(\omega),
$$

where $\omega$ is fixed as before.

We begin by introducing $v=u^{2^{*}-1} * \Gamma$, where $\Gamma$ is the positive fundamental solution of $\mathscr{L}$, that is, $\mathscr{L} \Gamma=-\delta$. According to [Folland 1975, Corollary 2.8], $v$ satisfies the equation $\mathscr{L} v=-u^{2^{*}-1}$. Since by (5-4) $u^{2^{*}-1}$ is in $\Gamma^{0, \beta}(\boldsymbol{G})$ for some $\beta \in(0,1)$ (and $u$ is compactly supported in $\boldsymbol{G}$ ), from [Folland 1975, Theorem 6.1] we have

$$
v \in \Gamma_{\text {loc }}^{2, \beta}(\boldsymbol{G}) .
$$

Therefore, if we let $w \stackrel{\text { def }}{=} u-v$, to prove (5-30) it is enough to show it for $w$, that is, to prove that $X w \in L^{\infty}(\omega)$. We notice that $w$ is $\mathscr{L}$-harmonic, that is, $\mathscr{L} w=0$ in $\Omega$.

From Theorem 5.6,

$$
u(g) \leq C d(g, \partial \Omega) \quad \text { for every } g \in \omega .
$$


Since we know that $u \in C^{\infty}(\bar{\Omega} \backslash \omega)$, we conclude that

$$
u(g) \leq C d(g, \partial \Omega) \quad \text { for every } g \in \Omega
$$

see Theorem 2.3. Fix a point $g \in \omega$. With $r=\operatorname{dist}(g, \partial \Omega) / 2$, consider the ball $B(g, r) \subset \bar{B}(g, r) \subset \Omega$. Applying the interior Schauder estimates in Theorem 2.2 to the $\mathscr{L}$-harmonic function $w-w(g)$, one has

$$
|X w(g)| \leq \frac{C}{r} \sup _{B(g, r)}(w-w(g)) .
$$

Note that (5-32) gives, for $g^{\prime} \in B(g, r)$,

$$
u\left(g^{\prime}\right) \leq C \operatorname{dist}\left(g^{\prime}, \partial \Omega\right) \leq C\left(d\left(g^{\prime}, g\right)+\operatorname{dist}(g, \partial \Omega)\right) \leq C r .
$$

Since $w=u-v$, in view of (5-34) and (5-32) one has, for $g^{\prime} \in B(g, r)$,

$$
\left|w\left(g^{\prime}\right)-w(g)\right| \leq\left(u\left(g^{\prime}\right)+u(g)\right)+\left|v\left(g^{\prime}\right)-v(g)\right| \leq C\left(r+\left|v\left(g^{\prime}\right)-v(g)\right|\right) .
$$

Finally, we observe that (5-31) implies that $v \in \mathscr{L}^{1, \infty}(\Omega)$ Applying Theorem 2.3 once more, we conclude that

$$
\left|v(g)-v\left(g^{\prime}\right)\right| \leq C d\left(g, g^{\prime}\right) \quad \text { for } g, g^{\prime} \in \Omega .
$$

Substitution of this information in (5-35) gives

$$
\sup _{B(g, r)}(w-w(g)) \leq C r .
$$

Combining the latter inequality with (5-33) brings the sought-for conclusion $X w \in$ $L^{\infty}(\omega)$. This finishes the proof of Theorem 5.10.

To end this section, we note that one can show the boundedness of the radial derivative of solutions of the Yamabe equation. This was done in [Garofalo and Vassilev 2000, Theorem 4.7], which we state below. The proof requires that $\boldsymbol{G}$ be of step 2 and the considered domain be $C^{\infty}$, bounded, connected, uniformly starlike with respect to one of its points, and satisfying (5-2) and (5-3).

Theorem 5.11. Let $\boldsymbol{G}$ be a Carnot group of step 2. Consider a $C^{\infty}$ connected, uniformly starlike, bounded open set $\Omega \subset \boldsymbol{G}$ satisfying (5-2) and (5-3). If $u$ is a weak solution of (1-4), then

$$
Z u \in L^{\infty}(\Omega) .
$$

5E. The estimates near the characteristic boundary for a domain in the Heisenberg group. Let $\Omega$ be a smooth domain in a Carnot group. Denote by $\Sigma$ its characteristic set with respect to the system $X=\left\{X_{1}, \ldots, X_{m}\right\}$, that is,

$$
\Sigma=\left\{g \in \partial \Omega \mid X_{j}(g) \in T_{g}(\partial \Omega), j=1, \ldots, m\right\} .
$$


Clearly, when the domain is bounded this is a compact subset of the boundary. In the next well-known theorem we denote by $N=\sum_{j=1}^{r} \operatorname{dim} V_{j}$ the topological dimension of $\boldsymbol{G}$. The symbol $H_{N-1}$ denotes the $(N-1)$-dimensional Hausdorff measure constructed using the Riemannian distance on $\boldsymbol{G}$.

Theorem 5.12. Let $\Omega \subset \boldsymbol{G}$ be a $C^{\infty}$ domain. One has

$$
H_{N-1}(\Sigma)=0 .
$$

This result is due to Derridj [1972]. A more refined version has been recently proved by Franchi and Wheeden [1997].

We now consider the Heisenberg group $\mathbb{\boxplus}^{n}$ and take $\Omega \subset \mathbb{H}^{n}$. We shall use the notation used until now, except that $U$ will be a sufficiently small neighborhood of $\Sigma$ - that is, $\Sigma \Subset U-$ and $\omega=U \cap \Omega$ as before. We also set $\Delta=\partial \Omega \cap U$. We recall the following Schauder estimates [Jerison 1981a]:

Theorem 5.13. Let $\Omega$ be a bounded $C^{\infty}$ domain in the Heisenberg group $\mathbb{H}^{n}$, and let $\varphi \in C_{0}^{\infty}\left(\mathbb{M}^{n}\right)$ be supported in a small neighborhood of a noncharacteristic point $g_{0} \in \partial \Omega$. Given $f \in \Gamma^{k, \alpha}(\bar{\Omega}), k \in \mathbb{N} \cup\{0\}, 0<\alpha<1$, for the unique solution $u$ to the Dirichlet problem for the Kohn sub-Laplacian

$$
\mathscr{L} u=f \quad \text { in } \Omega, \quad u=0 \quad \text { on } \partial \Omega,
$$

one has $\varphi u \in \Gamma^{k+2, \alpha}(\bar{\Omega})$.

Following the arguments in the preceding sections, we can prove the following:

Theorem 5.14. Let $\Omega$ be a smooth connected bounded open set in $\mathbb{H}^{n}$, satisfying the A-condition. Suppose that the convexity condition is satisfied in an interior neighborhood $\omega$ of its characteristic set. If $u$ is a weak nonnegative solution of the CR Yamabe equation, then the horizontal gradient of $u$ is bounded in $\Omega$. If in addition $\partial \Omega$ is uniformly starlike along $\Sigma$, then the radial derivative $Z u$ is bounded in $\Omega$.

Proof. As already mentioned, $u \in \Gamma^{0, \alpha}\left(\mathbb{\mathbb { H } ^ { n }}\right) \cap C^{\infty}(\Omega)$. From Theorem 5.13, by taking into account that $(Q+2) /(Q-2)>1$, we have $u \in \Gamma_{\text {loc }}^{2, \alpha}(\bar{\Omega} \backslash \Sigma)$. Now we can argue as in Theorem 5.10, which holds as long as we have Theorem 5.6 and Theorem 5.11, with the only difference that now $\partial \Omega$ should be replaced with $\Delta$. However, by doing this we see that $\partial \omega=\Delta \cup(\partial \omega \backslash \Delta)$, with $\partial \omega \backslash \Delta$ reaching $\partial \Omega$. There are two places where this is important:

(1) in Theorem 5.6, for (5-21);

(2) in Theorem 5.11, for the bound on $\partial \omega_{\lambda} \backslash \partial \Omega_{\lambda}$, in the proof's last paragraph.

We start with (1). The set $\Lambda=(\partial \omega \cap \bar{\Omega}) \backslash \Delta$ is at a fixed distance away from the characteristic set $\Sigma$. Therefore, for every $g_{0} \in \Lambda$, there exists $j \in\{1, \ldots, m\}$ such 
that $X_{j} \rho\left(g_{0}\right) \neq 0$. By continuity, the trajectories of $X_{j}$ fill a (sufficiently small) full neighborhood $V_{g_{0}}$ of $g_{0}$. This means that there exists $t_{0}=t\left(g_{0}\right)>0$ such that every $g \in \Omega \cap V_{g_{0}}$ can be written as $g_{1} \exp t X_{j}$ for some $g_{1} \in \partial \Omega \cap V_{g_{0}}$ and some $t \in\left(0, t_{0}\right)$. Using the uniform transversality of $X_{j}$ to $\partial \Omega$ in $\Omega \cap V_{g_{0}}$ and Taylor's formula, we infer the existence of $C=C\left(g_{0}\right)>0$ such that

$$
\left|R-\rho\left(g_{1} \exp t X_{j}\right)\right| \geq C|t|
$$

for every $g_{1} \in \partial \Omega \cap V_{g_{0}}$ and $0<t<t_{0}$. We now use that $u \in \Gamma_{\text {loc }}^{2, \alpha}(\bar{\Omega} \backslash \Sigma)$ to deduce the existence of a constant $C^{*}=C^{*}\left(u, g_{0}\right)>0$ such that

$$
u\left(g_{1} \exp t X_{j}\right) \leq C^{*}|t| \leq C^{*}|t|^{\alpha \sigma_{0}}
$$

for every $g_{1} \in \partial \Omega \cap V_{g_{0}}$ and $0<t<t_{0}$. The latter inequality and (5-39) allow us to conclude that (5-21) does hold in the set $\Omega \cap V_{g_{0}}$, for a constant depending on $u$ and $g_{0}$. By a finite-covering argument, we see that (5-21) continues to hold in the intersection of a small neighborhood of $\partial \Omega$ with $\Lambda$. We can thus separate from $\partial \Omega$. Once inside $\Omega$, we can use the $C^{\infty}$ smoothness of $u$ to conclude that (5-21) holds on the remaining portion of $\partial \omega \cap \Omega$ as well. This proves (1).

The embedding theorem 5.25 in [Folland 1975] implies that

$$
\Gamma_{\operatorname{loc}}^{2, \beta}(\boldsymbol{G}) \subset \Lambda_{\mathrm{loc}}^{1, \beta / 2}(\boldsymbol{G})=C_{\mathrm{loc}}^{1, \beta / 2}(\boldsymbol{G}),
$$

where the latter space denotes the standard Hölder class with respect to the Riemannian distance $d_{R}(\cdot, \cdot)$ on $\boldsymbol{G}$. The proof of (2) follows from the above embedding.

Remark 5.15. The previous theorem can be proved for any group of step 2, by requiring also that $u \in \Gamma_{\text {loc }}^{2, \alpha}(\bar{\Omega} \backslash \Sigma)$. With this assumption, we can also prove that

$$
\tilde{\eta} u \in L^{\infty}(\Omega),
$$

provided that $\tilde{\eta}$ is transversal to $\Sigma$. Here, $\eta \in V_{2}$ and $\tilde{\eta}$ is the corresponding leftinvariant vector field on a group $\boldsymbol{G}$ of step 2 .

We want to give an example of domains in groups of step 2 satisfying the assumptions of the preceding sections. Let $\boldsymbol{G}$ be a Carnot group of step 2. Using exponential coordinates, we define the function

$$
f_{\varepsilon}(g)=\left(\left(\varepsilon^{2}+|x(g)|^{2}\right)^{2}+16|y(g)|^{2}\right)^{1 / 4}, \quad \varepsilon \in \mathbb{R} .
$$

For $R>0$ and $\varepsilon \in \mathbb{R}$ with $\varepsilon^{2}<R^{2}$, consider the $C^{\infty}$ bounded open set

$$
\Omega_{R, \varepsilon}=\left\{g \in \boldsymbol{G} \mid f_{\varepsilon}(g)<R\right\} .
$$

When $\varepsilon=0$, it is clear that $\Omega_{R, \varepsilon}$ is nothing but a gauge pseudo-ball centered at the group identity $e$, except that the natural gauge was defined in (2-5) without the 
factor 16. Here, we have introduced such a factor for the purpose of keeping a consistent definition with the case of groups of Heisenberg type. For all practical purposes, the reader can ignore it and identify $f_{0}$ in (5-41) with (2-5). For $g \in \boldsymbol{G}$, we set $\Omega_{R, \varepsilon}(g)=\left\{h \in \boldsymbol{G} \mid f_{\varepsilon}\left(g^{-1} h\right)<R\right\}=g \Omega_{R, \varepsilon}$.

Theorem 5.16. Let $\boldsymbol{G}$ be a Carnot group of step 2. For every $\varepsilon \in \mathbb{R}$ with $\varepsilon^{2}<R^{2}$, the domain $\Omega_{R, \varepsilon}(g)$ satisfies the A-condition, the convexity condition (globally), and is uniformly starlike.

For the proof of this theorem see [Garofalo and Vassilev 2000].

\section{Acknowledgments}

Apart from small changes and the addition of some updates in the references, this paper is part of the author's doctoral dissertation at Purdue University, 2000. The author would like to thank his advisor Professor Nicola Garofalo for his guidance, support, and encouragement.

The author would also like to acknowledge the careful reading of the anonymous referee and thank him/her for the comments, which improved the reading of the paper.

\section{References}

[Aubin 1976a] T. Aubin, "Équations différentielles non linéaires et problème de Yamabe concernant la courbure scalaire", J. Math. Pures Appl. (9) 55:3 (1976), 269-296. MR 55 \#4288 Zbl 0336.53033

[Aubin 1976b] T. Aubin, "Problèmes isopérimétriques et espaces de Sobolev", J. Differential Geometry 11:4 (1976), 573-598. MR 56 \#6711 Zbl 0371.46011

[Bahri and Coron 1988] A. Bahri and J.-M. Coron, "On a nonlinear elliptic equation involving the critical Sobolev exponent: the effect of the topology of the domain", Comm. Pure Appl. Math. 41:3 (1988), 253-294. MR 89c:35053 Zbl 0649.35033

[Bony 1969] J.-M. Bony, "Principe du maximum, inégalite de Harnack et unicité du problème de Cauchy pour les opérateurs elliptiques dégénérés", Ann. Inst. Fourier (Grenoble) 19:fasc. 1 (1969), 277-304 xii. MR 41 \#7486 Zbl 0176.09703

[Brezis 1986] H. Brezis, "Elliptic equations with limiting Sobolev exponents-the impact of topology”, Comm. Pure Appl. Math. 39:S, suppl. (1986), S17-S39. MR 87k:58272 Zbl 0601.35043

[Brézis and Kato 1979] H. Brézis and T. Kato, "Remarks on the Schrödinger operator with singular complex potentials", J. Math. Pures et Appliquées. (9) 58:2 (1979), 137-151. MR 80i:35135 Zbl 0408.35025

[Brézis and Nirenberg 1983] H. Brézis and L. Nirenberg, "Positive solutions of nonlinear elliptic equations involving critical Sobolev exponents", Comm. Pure Appl. Math. 36:4 (1983), 437-477. MR 84h:35059 Zbl 0541.35029

[Capogna and Garofalo 1998] L. Capogna and N. Garofalo, "Boundary behavior of nonnegative solutions of subelliptic equations in NTA domains for Carnot-Carathéodory metrics", J. Fourier Anal. Appl. 4:4-5 (1998), 403-432. MR 2000k:35056 Zbl 0926.35043 
[Capogna et al. 1993] L. Capogna, D. Danielli, and N. Garofalo, "An embedding theorem and the Harnack inequality for nonlinear subelliptic equations", Comm. Partial Differential Equations 18:910 (1993), 1765-1794. MR 94j:35038 Zbl 0802.35024

[Capogna et al. 1998] L. Capogna, N. Garofalo, and D.-M. Nhieu, "A version of a theorem of Dahlberg for the subelliptic Dirichlet problem", Math. Research Letters 5:4 (1998), 541-549. MR 2000k:35057 Zbl 0934.22017

[Citti and Uguzzoni 2001] G. Citti and F. Uguzzoni, "Critical semilinear equations on the Heisenberg group: the effect of the topology of the domain", Nonlinear Anal. 46:3, Ser. A: Theory Methods (2001), 399-417. MR 2002g:35064 Zbl 01660651

[Danielli and Garofalo 1998] D. Danielli and N. Garofalo, "Geometric properties of solutions to subelliptic equations in nilpotent Lie groups", pp. 89-105 in Reaction diffusion systems (Trieste, 1995), edited by G. Caristi and E. Mitidieri, Lecture Notes in Pure and Appl. Math. 194, Dekker, New York, 1998. MR 99a:22015 Zbl 0903.58048

[Derridj 1971] M. Derridj, “Un problème aux limites pour une classe d'opérateurs du second ordre hypoelliptiques”, Annales de l'Institut Fourier (Grenoble) 21:4 (1971), 99-148. MR 58 \#29139 Zbl 0215.45405

[Derridj 1972] M. Derridj, "Sur un théorème de traces", Ann. Inst. Fourier (Grenoble) 22:2 (1972), 73-83. MR 49 \#7755 Zbl 0231.46076

[Folland 1975] G. B. Folland, "Subelliptic estimates and function spaces on nilpotent Lie groups", Ark. Mat. 13:2 (1975), 161-207. MR 58 \#13215 Zbl 0312.35026

[Folland and Stein 1974] G. B. Folland and E. M. Stein, "Estimates for the $\bar{\partial}_{b}$ complex and analysis on the Heisenberg group", Comm. Pure Appl. Math. 27 (1974), 429-522. MR 51 \#3719 Zbl 0293.35012

[Franchi and Wheeden 1997] B. Franchi and R. L. Wheeden, "Compensation couples and isoperimetric estimates for vector fields”, Colloquium Mathematicum 74:1 (1997), 9-27. MR 98g:46042 Zbl 0915.46028

[Franchi et al. 1994] B. Franchi, C. E. Gutiérrez, and R. L. Wheeden, "Weighted Sobolev-Poincaré inequalities for Grushin type operators", Comm. Partial Differential Equations 19:3-4 (1994), 523604. MR 96h:26019 Zbl 0822.46032

[Gamara 2001] N. Gamara, "The CR Yamabe conjecture - the case $n=1$ ", J. Eur. Math. Soc. (JEMS) 3:2 (2001), 105-137. MR 2003d:32040a Zbl 0988.53013

[Gamara and Yacoub 2001] N. Gamara and R. Yacoub, "CR Yamabe conjecture - the conformally flat case", Pacific J. Math. 201:1 (2001), 121-175. MR 2003d:32040b Zbl 1054.32020

[Garofalo and Nhieu 1996] N. Garofalo and D.-M. Nhieu, "Isoperimetric and Sobolev inequalities for Carnot-Carathéodory spaces and the existence of minimal surfaces", Comm. Pure Appl. Math. 49:10 (1996), 1081-1144. MR 97i:58032 Zbl 0880.35032

[Garofalo and Nhieu 1998] N. Garofalo and D.-M. Nhieu, "Lipschitz continuity, global smooth approximations and extension theorems for Sobolev functions in Carnot-Carathéodory spaces", $J$. Anal. Math. 74 (1998), 67-97. MR 2000i:46025 Zbl 0906.46026

[Garofalo and Vassilev 2000] N. Garofalo and D. Vassilev, "Regularity near the characteristic set in the non-linear Dirichlet problem and conformal geometry of sub-Laplacians on Carnot groups", Math. Ann. 318:3 (2000), 453-516. MR 2001j:35042 Zbl pre01545454

[Garofalo and Vassilev 2001] N. Garofalo and D. Vassilev, "Symmetry properties of positive entire solutions of Yamabe-type equations on groups of Heisenberg type", Duke Math. J. 106:3 (2001), 411-448. MR 2002j:35075 Zbl 1012.35014 
[Gilbarg and Trudinger 1983] D. Gilbarg and N. S. Trudinger, Elliptic partial differential equations of second order, Second ed., Grundlehren der Mathematischen Wissenschaften 224, SpringerVerlag, Berlin, 1983. MR 86c:35035 Zbl 0562.35001

[Holopainen 1992] I. Holopainen, "Positive solutions of quasilinear elliptic equations on Riemannian manifolds", Proc. London Mathematical Society (3) 65:3 (1992), 651-672. MR 94d:58161 Zbl 0739.53030

[Holopainen and Rickman 1992] I. Holopainen and S. Rickman, "Quasiregular mappings of the Heisenberg group”, Math. Ann. 294:4 (1992), 625-643. MR 93m:30031 Zbl 0754.30018

[Hörmander 1967] L. Hörmander, "Hypoelliptic second order differential equations", Acta Math. 119 (1967), 147-171. MR 36 \#5526 Zbl 0156.10701

[Jerison 1981a] D. S. Jerison, "The Dirichlet problem for the Kohn Laplacian on the Heisenberg group. I”, J. Funct. Anal. 43:1 (1981), 97-142. MR 83c:58081a Zbl 0493.58021

[Jerison 1981b] D. S. Jerison, "The Dirichlet problem for the Kohn Laplacian on the Heisenberg group. II”, J. Funct. Anal. 43:2 (1981), 224-257. MR 83c:58081b Zbl 0493.58022

[Jerison and Lee 1984] D. Jerison and J. M. Lee, “A subelliptic, nonlinear eigenvalue problem and scalar curvature on CR manifolds", pp. 57-63 in Microlocal analysis (Boulder, CO, 1983)), edited by M. S. Baouendi et al., Contemp. Math. 27, Amer. Math. Soc., Providence, RI, 1984. MR 85i:58122 Zbl 0577.53035

[Jerison and Lee 1987] D. Jerison and J. M. Lee, "The Yamabe problem on CR manifolds", J. Differential Geom. 25:2 (1987), 167-197. MR 88i:58162 Zbl 0661.32026

[Jerison and Lee 1988] D. Jerison and J. M. Lee, "Extremals for the Sobolev inequality on the Heisenberg group and the CR Yamabe problem", J. American Math. Society 1:1 (1988), 1-13. MR 89b:53063 Zbl 0634.32016

[Jerison and Lee 1989] D. Jerison and J. M. Lee, "Intrinsic CR normal coordinates and the CR Yamabe problem”, J. Differential Geom. 29:2 (1989), 303-343. MR 90h:58083 Zbl 0671.32016

[Krantz 1982] S. G. Krantz, "Lipschitz spaces on stratified groups", Trans. Amer. Math. Soc. 269:1 (1982), 39-66. MR 83c:22015 Zbl 0529.22006

[Lanconelli and Uguzzoni 1998] E. Lanconelli and F. Uguzzoni, "Asymptotic behavior and nonexistence theorems for semilinear Dirichlet problems involving critical exponent on unbounded domains of the Heisenberg group", Boll. Unione Mat. Ital. Sez. B Artic. Ric. Mat. (8) 1:1 (1998), 139-168. MR 99d:35058 Zbl 0902.22006

[Lee and Parker 1987] J. M. Lee and T. H. Parker, "The Yamabe problem", Bull. Amer. Math. Soc. (N.S.) 17:1 (1987), 37-91. MR 88f:53001 Zbl 0633.53062

[Lions 1984a] P.-L. Lions, "The concentration-compactness principle in the calculus of variations. The locally compact case. I”, Ann. Inst. H. Poincaré Anal. Non Linéaire 1:2 (1984), 109-145. MR 87e:49035a Zbl 0541.49009

[Lions 1984b] P.-L. Lions, "The concentration-compactness principle in the calculus of variations. The locally compact case. II", Ann. Inst. H. Poincaré Anal. Non Linéaire 1:4 (1984), 223-283. MR 87e:49035b Zbl 0704.49004

[Lions 1985a] P.-L. Lions, "The concentration-compactness principle in the calculus of variations. The limit case. I", Rev. Mat. Iberoamericana 1:1 (1985), 145-201. MR 87c:49007 Zbl 0704.49005

[Lions 1985b] P.-L. Lions, "The concentration-compactness principle in the calculus of variations. The limit case. II", Rev. Mat. Iberoamericana 1:2 (1985), 45-121. MR 87j:49012 Zbl 0704.49006

[Moser 1961] J. Moser, "On Harnack's theorem for elliptic differential equations", Comm. Pure Appl. Math. 14 (1961), 577-591. MR 28 \#2356 Zbl 0111.09302 
[Nagel et al. 1985] A. Nagel, E. M. Stein, and S. Wainger, "Balls and metrics defined by vector fields, I: Basic properties”, Acta Math. 155:1-2 (1985), 103-147. MR 86k:46049 Zbl 0578.32044

[Schoen 1984] R. Schoen, "Conformal deformation of a Riemannian metric to constant scalar curvature”, J. Differential Geom. 20:2 (1984), 479-495. MR 86i:58137 Zbl 0576.53028

[Serrin 1964] J. Serrin, "Local behavior of solutions of quasi-linear equations", Acta Math. 111 (1964), 247-302. MR 30 \#337 Zbl 0128.09101

[Struwe 1990] M. Struwe, Variational methods: Applications to nonlinear partial differential equations and Hamiltonian systems, Springer, Berlin, 1990. MR 92b:49002 Zbl 0746.49010

[Talenti 1976] G. Talenti, "Best constant in Sobolev inequality", Ann. Mat. Pura Appl. (4) 110 (1976), 353-372. MR 57 \#3846 Zbl 0353.46018

[Tanaka 1975] N. Tanaka, A differential geometric study on strongly pseudo-convex manifolds, Kinokuniya, Tokyo, 1975. MR 53 \#3361 Zbl 0331.53025

[Trudinger 1968] N. S. Trudinger, "Remarks concerning the conformal deformation of Riemannian structures on compact manifolds", Ann. Scuola Norm. Sup. Pisa (3) 22 (1968), 265-274. MR 39 \#2093 Zbl 0159.23801

[Xu 1990] C. J. Xu, "Subelliptic variational problems", Bull. Soc. Math. France 118:2 (1990), 147169. MR 92b:49008 Zbl 0717.49004

[Yamabe 1960] H. Yamabe, "On a deformation of Riemannian structures on compact manifolds", Osaka Math. J. 12 (1960), 21-37. MR 23 \#A2847 Zbl 0096.37201

Received September 14, 2004. Revised August 31, 2005.

Dimiter VASSILEV

UNIVERSITY OF CALIFORNIA, RIVERSIDE

RIVERSIDE, CA 92521

UNITED STATES

dvassilev@math.ucr.edu 